\title{
Analysis of Photocatalytic Nitrogen Fixation on Rutile $\mathrm{TiO}_{2}(\mathbf{1 1 0})$
}

\author{
Benjamin M. Comer and Andrew J. Medford* \\ School of Chemical \&3 Biomolecular Engineering, Georgia Institute of Technology, 311 Ferst \\ Dr NW, Atlanta, GA, 30318 \\ E-mail: andrew.medford@chbe.gatech.edu \\ Phone: +1 (404) 385-5531
}

\begin{abstract}
Photocatalytic nitrogen fixation provides a promising route to produce reactive nitrogen compounds at benign conditions. Titania has been reported as an active photocatalyst for reduction of dinitrogen to ammonia; however there is little fundamental understanding of how this process occurs. In this work the rutile (110) model surface is hypothesized to be the active site, and a computational model based on the Bayesian error estimation functional (BEEF-vdW) and computational hydrogen electrode is applied in order to analyze the expected dinitrogen coverage at the surface as well as the overpotentials for electrochemical reduction and oxidation. This is the first application of computational techniques to photocatalytic nitrogen fixation, and the results indicate that the thermodynamic limiting potential for nitrogen reduction on rutile (110) is considerably higher than the conduction band edge of rutile $\mathrm{TiO}_{2}$, even at oxygen vacancies and iron substitutions. This work provides strong evidence against the most commonly reported experimental hypotheses, and indicates that rutile (110) is unlikely to be the relevant surface for nitrogen reduction. However, the limiting potential for nitrogen oxidation on rutile (110) is significantly lower, indicating
\end{abstract}


that oxidative pathways may be relevant on rutile (110). These findings suggest that photocatalytic dinitrogen fixation may occur via a complex balance of oxidative and reductive processes.

\section{Keywords}

density functional theory, uncertainty analysis, nitrogen reduction, nitrogen oxidation, nitrogen fixation

\section{Introduction}

The photocatalytic reduction of atmospheric nitrogen was first proposed in the soil science community, ${ }^{1}$ and was later demonstrated under photocatalytic conditions by Schrauzer and Guth. ${ }^{2,3}$ These efforts spurred numerous studies on photocatalytic nitrogen fixation by titania catalysts ${ }^{4-10}$ that resulted in a variety of interesting observations. It was shown that dinitrogen can be converted to ammonia under both aqueous conditions ${ }^{5,9}$ and humidified air, ${ }^{2,3}$ with higher rates observed in gas-phase conditions. ${ }^{7}$ Further, the titania photocatalysts selectively reduce nitrogen to ammonia in most studies, ${ }^{2,3,5-7,9}$ although some work indicates that oxidation to nitrates occurs, ${ }^{4,8}$ and other authors observed no photocatalytic nitrogen fixation activity. ${ }^{11-14}$ In addition, the presence of the rutile crystal phase and iron dopants were found to be critical for enhancing nitrogen reduction activity. ${ }^{2,3,5,6}$ The iron dopants have been proposed to promote the formation of rutile domains, ${ }^{2}$ and/or enhance separation of charge carriers by acting as an electron sink; ${ }^{6}$ while the exact role is not known, the prevailing hypothesis is that Fe is not part of the active site based on the observation that excess Fe content reduces catalytic activity. ${ }^{6}$ More recently, the solar-to-ammonia efficiency was measured to be around $0.02 \%$ for a pure titania sample, and the authors hypothesize that the reaction occurs via direct $\mathrm{N}-\mathrm{N}$ bond scission at a rutile (110) bridging oxygen vacancy (O-br) active site. ${ }^{9}$ Despite the long history of photocatalytic nitrogen fixation on titania 
catalysts and numerous experimental results, there is little fundamental understanding of the molecular-scale processes that underly this important and potentially transformative photocatalytic process. In this work we investigate the hypothesis that rutile (110) (Figure 1 ) is the active surface for photocatalytic nitrogen fixation by examining the pristine rutile (110) surface, the defected (110) surface containing an oxygen vacancy, an iron substitution at the (110) surface, and oxidative processes on the pristine (110) surface.

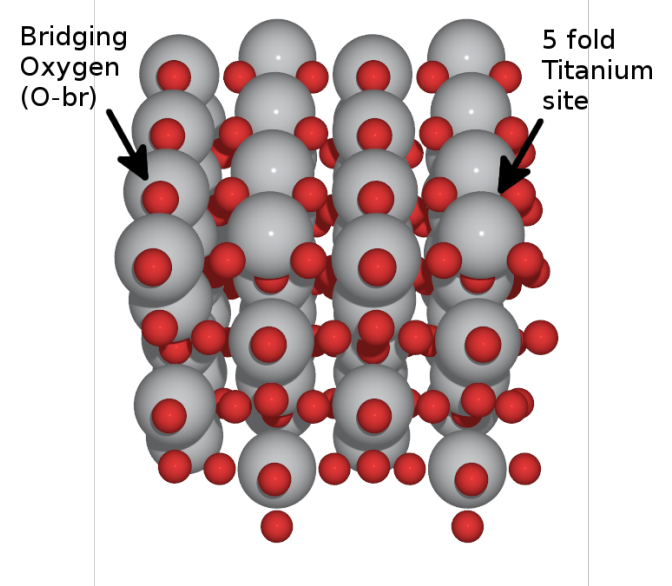

Figure 1: Illustration of $\mathrm{TiO}_{2}(110)$

The rutile $\mathrm{TiO}_{2}$ (110) surface has been well-studied both experimentally ${ }^{15-20}$ and computationally; ${ }^{21-24}$ however, there have been relatively few computational studies of the interactions of rutile $\mathrm{TiO}_{2}$ surfaces with nitrogen-containing compounds. ${ }^{21-23,25,26}$ Two comprehensive computational studies on nitrogen oxides were performed by Stodt et al. ${ }^{21}$ and Sorescu et al. ${ }^{22}$ These studies examined $\mathrm{N}_{x} \mathrm{O}_{y}$ compounds on $\mathrm{TiO}_{2}$ (110) surfaces both experimentally under ultrahigh vacuum (UHV) conditions and theoretically using density functional theory (DFT) at the generalized gradient approximation (GGA) ${ }^{22}$ and hybrid ${ }^{21}$ levels of theory. This work showed that DFT is able to accurately obtain the vibrational frequencies of intermediates on the surface and is consistent with UHV experiments. In addition, Cheng et al. ${ }^{23}$ examined the adsorption of $\mathrm{NH}_{3}$ (ammonia), $\mathrm{NH}_{2}$, and $\mathrm{H}$ on clean rutile (110) surfaces using DFT. They concluded that $\mathrm{NH}_{3}$ and $\mathrm{NH}_{2}$ adsorb at the 5-fold titanium 
site, whereas hydrogen could bind at the bridging oxygens or the in-plane oxygen atoms. Several studies have also investigated the binding of ammonia and other nitrogen-containing species on anatase ${ }^{27-31}$ and monoclinic ${ }^{32}$ polymorphs of $\mathrm{TiO}_{2}$. Very recently Xie et. al. used DFT to calculate the energetics of nitric oxide reduction on $\mathrm{TiO}_{2},{ }^{26}$ and Höskuldsson et. al. utilized GGA DFT to screen rutile oxides for electrochemical ammonia synthesis, and found a relatively large limiting potential of ca. $2 \mathrm{~V}$ for the (110) surface of rutile $\mathrm{TiO}_{2} \cdot{ }^{25}$

In contrast to the relatively low number of computational studies there have been many experimental studies of nitrogen compounds on rutile surfaces. Yates and colleagues conducted several studies of $\mathrm{NO}_{\mathrm{x}}$ molecules under UHV conditions over rutile $\mathrm{TiO}_{2}(110) .{ }^{17-19}$ Their early work showed that NO adsorbs and decomposes to form $\mathrm{N}_{2} \mathrm{O}$ on reduced surfaces, ${ }^{17}$ and a follow up study involved photochemical activation of NO on the surface that resulted in the production of $\mathrm{N}_{2} \mathrm{O}$, even with photon energies below the band gap. ${ }^{18}$ When the surface is dosed with $\mathrm{N}_{2} \mathrm{O}$ and exposed to photons at high coverage, $\mathrm{N}_{2} \mathrm{O}$ is desorbed from the surface, while at low coverages $\mathrm{N}_{2}$ is observed. ${ }^{19}$ Much of this was also seen in the recent examination by Kim and colleagues with the additional insight that surface oxygen vacancies play an important role. ${ }^{33,34}$ Furthermore, numerous applied studies focusing on nitrogen doped titania, ${ }^{35} \mathrm{TiO}_{2}$ as an ammonia sensor ${ }^{36,37}$ or in the selective catalytic reduction (SCR) process ${ }^{38-41}$ provide insight into the interaction of titania with nitrogen-containing compounds. Although $\mathrm{TiO}_{2}$ is typically not considered to be the active phase for the SCR reaction, ${ }^{42}$ it is interesting to note a small body of work on the "photo-SCR" where anatase $\mathrm{TiO}_{2}$ has been proposed as an active and selective catalyst. ${ }^{31,43-49}$ These studies provide useful context for the photocatalytic nitrogen fixation as they involve the interaction of both oxidized and reduced nitrogen species with titania surfaces.

In addition to nitrogen chemistry, the interaction of titania surfaces with water will play a role in aqueous or humidified environments. Water adsorption on $\mathrm{TiO}_{2}$ is a topic of extensive research and debate, ${ }^{15,50-58}$ and there are a great variety of theoretical results regarding the competition between dissociative ${ }^{55,58}$ and molecular adsorption ${ }^{56,57,59}$ on pristine surfaces, 
although a clear consensus has not emerged. Analyses including the effects of slab thickness ${ }^{51}$ and method ${ }^{52}$ support the two modes being nearly degenerate at monolayer coverages, with partial dissociation having a slightly higher energy. This viewpoint is consistent with recent experimental studies observing dissociated water on pristine surfaces. ${ }^{16}$ Experimental studies from the mid 1990's to the mid 2000's show that on a reduced surface (one containing vacancies at the bridging oxygens) water tends to dissociate to form two hydroxyl groups on the surface, thereby filling the vacancy, ${ }^{57,60-62}$ while on a pristine surface water adsorbs molecularly at the 5 -fold Ti site. ${ }^{63}$ As previously noted there has been one recent report showing partial dissociation of water on the pristine surface. ${ }^{16}$

Several other factors have been observed to play an important role in aqueous $\mathrm{TiO}_{2}$ photochemistry. The formation of hydroxyl radicals is known to occur in both rutile and anatase, ${ }^{64,65}$ and these radicals participate in a number of oxidation reactions. ${ }^{64}$ Furthermore, the influence of both surface and bulk defects such as oxygen vacancies can enhance adsorption and influence photocatalytic activity. ${ }^{66}$ The field of $\mathrm{TiO}_{2}$ photocatalysis and surface science is vast, and a thorough review is beyond the scope of this work; the reader is referred to published reports and review articles to gain a more complete view. ${ }^{15,20,53,54,67}$

In this work we present an initial computational investigation of photocatalytic nitrogen reduction and oxidation on the rutile $\mathrm{TiO}_{2}(110)$ surface. The rutile (110) surface is hypothesized to be the active surface due to the fact that photocatalytic nitrogen fixation rates have been observed to correlate with the amount of rutile in $\mathrm{TiO}_{2}$ samples; ${ }^{7}$ the (110) surface is the lowest energy surface on rutile and is likely to provide a model for other rutile surfaces. Furthermore, the rutile (110) surface has been explicitly hypothesized to be the active surface in recent experimental work. ${ }^{9}$ The availability of adsorbed nitrogen on rutile (110) is investigated under aqueous and gas-phase conditions, and the thermodynamic stability of adsorbed intermediates for nitrogen reduction and oxidation are investigated. We utilize ab initio thermodynamics ${ }^{68,69}$ and the simple but effective "computational hydrogen electrode" (CHE) approach ${ }^{70}$ that has proven useful in understanding the oxygen reduction, ${ }^{71}$ 
oxygen evolution, ${ }^{72} \mathrm{CO}_{2}$ reduction, ${ }^{73}$ and nitrogen reduction ${ }^{74}$ reactions. Furthermore, the Bayesian error estimation functional (BEEF-vdW) error estimation functional ${ }^{75}$ is utilized to asses the robustness of the conclusions. The results indicate that nitrogen adsorption is more favorable under gas-phase conditions, providing a simple explanation for the improved experimental performance. However, the computational evidence refutes the hypothesis that the rutile (110) surface is the active site for nitrogen reduction due to the low stability of adsorbed $\mathrm{N}_{2} \mathrm{H}_{\mathrm{x}}$ and $\mathrm{NH}_{\mathrm{x}}$ compounds at the pristine surface and at oxygen vacancy and Fe-substitution sites. In contrast, computational results indicate that nitrogen oxidation to NO is a thermodynamically viable fixation route on rutile (110), and it is hypothesized that the N-N bond is dissociated through an oxidative process and the NO intermediate is subsequently reduced.

\section{Results and Discussion}

The study of photocatalytic nitrogen fixation on $\mathrm{TiO}_{2}$ is carried out using DFT calculations to provide molecular-scale insight into the thermochemistry of adsorbed intermediate states. Computational investigations of photocatalysis on $\mathrm{TiO}_{2}$ are difficult due to the complex nature of photocatalytic interfaces ${ }^{70,76}$ and the electronic structure of $\mathrm{TiO}_{2} \cdot{ }^{67,77}$ In this work we utilize simple models to establish the feasibility of various hypotheses. The energetics of adsorbed species are computed under ideal gas-phase conditions (e.g. solvent effects, electric field effects, and coverage effects are neglected) and it is assumed that excitation and charge transport are decoupled from the electrochemical reactions, enabling the use of the $\mathrm{CHE}$ approach; ${ }^{70,71,73,76}$ all voltages are relative to $\mathrm{CHE}$ (equivalent to RHE) unless otherwise noted. To treat the electronic structure the generalized gradient approximation (GGA) with van der Waals (vdW) level of theory is used. The use of the Bayesian error

estimation functional ${ }^{75}$ (BEEF-vdW) provides error estimates that quantify uncertainty in order to assess the sensitivity of conclusions to the error due to the GGA approximation; more 
details are provided in the Methods section. This approach is used to test four hypotheses, as discussed in the following sections: i) photocatalytic nitrogen fixation rates are greater in the gas phase than the aqueous phase ii) rutile (110) is an active surface for nitrogen reduction iii) oxygen vacancies or Fe substititions on rutile (110) are active sites for nitrogen reduction iv) rutile (110) is an active surface for nitrogen oxidation and subsequent NO reduction.

\section{Nitrogen adsorption under gas and aqueous environments}

The literature contains examples of experiments done in aqueous solution ${ }^{5,9}$ and humidified air. $^{2,3}$ Of these, humidified air generally has a higher rate. ${ }^{7}$ To better understand the difference in performance under aqueous and gas-phase conditions ab-initio thermodynamics ${ }^{68}$ have been used to compute surface phase diagrams for rutile (110) as a function of water and nitrogen chemical potentials. Fig. 2a shows the surface free energy (top) and surface coverage (middle) of the reactants available at ambient gas-phase conditions $\left(\mathrm{H}_{2} \mathrm{O}, \mathrm{N}_{2}, \mathrm{O}_{2}\right.$, $\mathrm{OH}$ ) (a similar analysis including all reactants, products and intermediates is available in the Supporting Information). The results show that the bare $\mathrm{TiO}_{2}$ surface is dominant at gasphase conditions. The ensemble of energies from the BEEF-vdW functional can be exploited to evaluate the sensitivity of these coverages to DFT error. The probability analysis (Fig 2a, bottom) suggests that there is a very low probability of having appreciable $\mathrm{N}_{2}$ coverage at $100 \% \mathrm{RH}$, but under dry conditions there is a probability of $\approx 10 \%$ of having a $\mathrm{N}_{2}$ coverage $>0.25 \mathrm{ML}$. This suggests that arid environments may favor $\mathrm{N}_{2}$ adsorption, though it is noted that water also acts as a proton source so nitrogen reduction cannot occur if the humidity is too low. One interpretation of the surface coverage probability is that the BEEF-vdW functional is not sufficiently accurate to precisely determine the surface coverage, though the bare surface is the most likely. Another perspective is to view this as a sensitivity analysis of coverage with respect to binding energies of competing surface species. From this view, the results indicate that adsorption sites with slightly higher relative $\mathrm{N}_{2}$ adsorption are likely to have a significant $\mathrm{N}_{2}$ coverage under gas-phase conditions. This could include defect sites 

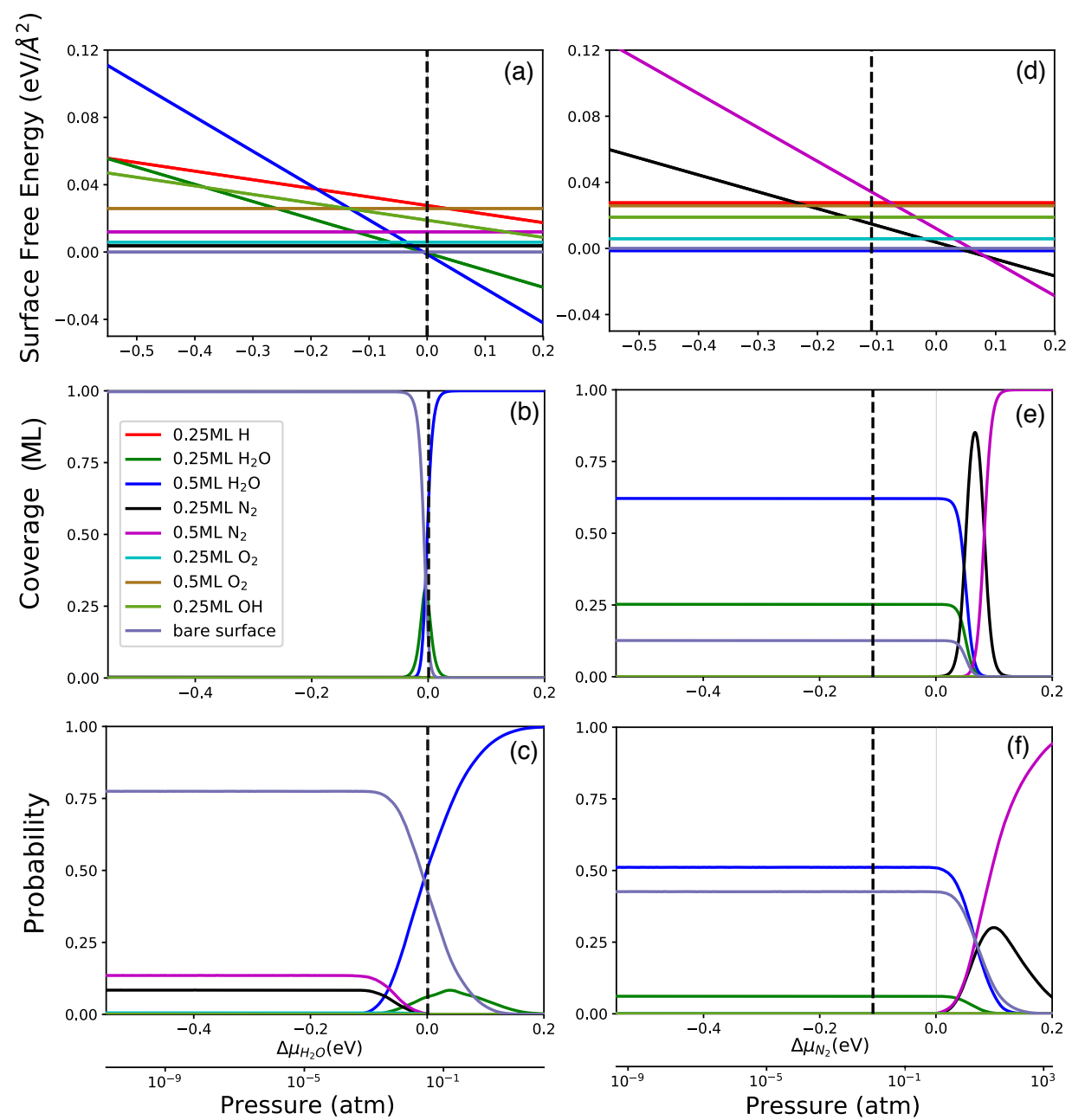

Figure 2: Surface free energy (a,d), coverage (b,e), and coverage probability (c,f) for $\mathrm{H}_{2} \mathrm{O}$, $\mathrm{N}_{2}, \mathrm{O}_{2}$, and $\mathrm{OH}$ as a function of $\mathrm{H}_{2} \mathrm{O}$ (a-c) and $\mathrm{N}_{2}$ (d-f) chemical potentials. The relevant water potential under gas-phase conditions (0.035 atm) and nitrogen potential under aqueous conditions (0.012 atm) are shown by dashed lines in (a-c) and (d-f) respectively. Graphs a-c use constant $\mathrm{N}_{2}$ chemical potential set at atmospheric pressure of $\mathrm{N}_{2}(0.8 \mathrm{~atm})$ and d-f use constant water chemical potential set at $100 \%$ relative humidity of $\mathrm{H}_{2} \mathrm{O}$. Nitrogen pressure at aqueous conditions is estimated using Henry's law. The probability of various coverages $(\mathrm{c}, \mathrm{f})$ given the uncertainty in the BEEF-vdW functional is calculated using Eq. 15. 
or other facets, although the site must have both stronger absolute binding energies and stronger relative adsorption of $\mathrm{N}_{2}$ vs. $\mathrm{H}_{2} \mathrm{O}$. There are likely sites that satisfy this criterion, but their prevalence must also be considered. Regardless of the interpretation, the conclusion is that nitrogen coverages are expected to be relatively low on rutile $\mathrm{TiO}_{2}$ (110) which is unsurprising given the inert nature of $\mathrm{N}_{2}$.

The surface phase diagrams under aqueous conditions (2b) indicate that water will be the dominant surface species for a wide range of $\mathrm{N}_{2}$ pressures, with adsorbed $\mathrm{N}_{2}$ becoming dominant at approximately 100 atm. In this case the probability analysis indicates that although the surface coverage of water/hydroxyl is not well-determined by the BEEF-vdW functional, the probability of a significant $\mathrm{N}_{2}$ coverage is negligible at aqueous conditions. Interestingly, several reports have shown appreciable nitrogen fixation rates under aqueous conditions. $^{5,9}$ The fact that competitive nitrogen adsorption is not favored under aqueous conditions indicates that highly reactive surface groups or defect sites with higher $\mathrm{N}_{2}$ binding energy but low stability may play a role. The finding that nitrogen adsorption is more favorable in the gas phase suggests that the enhanced photocatalytic activity under gas phase conditions is due to the improved ability to adsorb $\mathrm{N}_{2}$ in the absence of water. This intuitive result corroborates previous findings indicating that a key challenge in photocatalytic nitrogen fixation is getting $\mathrm{N}_{2}$ to adsorb, ${ }^{7,78,79}$ and qualitatively explains the correlation be-

tween $\mathrm{N}_{2}$ pressure and photocatalytic nitrogen reduction activity. ${ }^{7,80}$ The issue of nitrogen adsorption must be addressed in any photo(electro)catalytic system regardless of reaction mechanism, and will be a fundamental problem for low pressure nitrogen fixation processes.

\section{Thermochemistry of nitrogen reduction}

The electrochemical nitrogen reduction reaction converts nitrogen to ammonia and occurs at an equilibrium potential of $0.05 \mathrm{~V}$ vs. RHE (Fig. 3). The proximity of this redox couple to the hydrogen evolution reaction $\left(\mathrm{H}^{+} / \mathrm{H}_{2}\right.$ at $\left.0 \mathrm{~V}\right)$ makes selective reduction of nitrogen to ammonia challenging. ${ }^{74,82,83}$ Furthermore, the alignment of the rutile $\mathrm{TiO}_{2}$ conduction band 


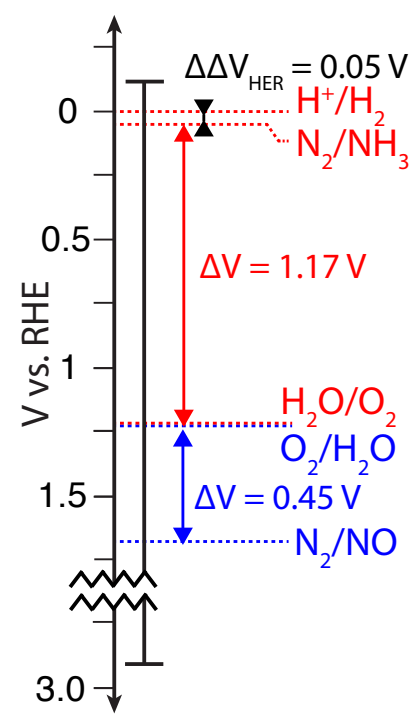

Figure 3: Redox couples for nitrogen reduction (red) and oxidation (blue) with required driving force $(\Delta V) .{ }^{10}$ The difference between hydrogen evolution and nitrogen reduction $\left(\Delta \Delta \mathrm{V}_{\mathrm{HER}}\right)$ is shown to highlight the selectivity challenge for nitrogen reduction. Band edges for rutile $\mathrm{TiO}_{2}$ were obtained from literature ${ }^{81}$ and are shown (black bar) for reference (note scale break at $1.75 \mathrm{~V}$ ).

edge (Fig. 3) indicates that the available overpotential for nitrogen reduction under photocatalytic conditions is relatively low $(<0.15 \mathrm{~V})$. Nonetheless, selective nitrogen reduction has been observed on $\mathrm{TiO}_{2}$ photocatalysts, ${ }^{2,3,5,7}$ indicating that $\mathrm{TiO}_{2}$ is capable of dissociating the strong $\mathrm{N}-\mathrm{N}$ bond more easily than the much weaker $\mathrm{H}-\mathrm{H}$ bond. This suggests that identification of the active site for photocatalytic nitrogen reduction may enable the development of improved nitrogen reduction electrocatalysts. In this section we hypothesize that the active site is pristine rutile (110), and examine the binding free energies of intermediates for the dissociative and associative nitrogen reduction pathways.

The mechanism for thermocatalytic nitrogen reduction (i.e. Haber-Bosch catalysis) is established as a dissociative mechanism in which the first step is scission of the N-N bond, followed by hydrogenation of adsorbed mono-nitrogen; ${ }^{84-87}$ the electrochemical equivalent is shown in equations $1-6$. Calculations of $\mathrm{N}-\mathrm{N}$ scission on rutile oxides shows that they follow "ideal" scaling, suggesting that the activation barrier for nitrogen dissociation may be low, ${ }^{88}$ hence we first investigate the dissociative mechanism. Figure 4 shows the free 
energy diagram for the dissociative mechanism at standard temperature and pressure at the equilibrium potential. The dissociation energy of $\mathrm{N}_{2}$ is remarkably high $(>8.5 \mathrm{eV})$, indicating that this route is not remotely thermodynamically feasible on rutile (110). This extreme barrier is not sensitive to the BEEF-vdW exchange-correlation approximation, and is expected to be prohibitively large even in the presence of solvent stabilization since each adsorbed $\mathrm{N}^{*}$ would need to be stabilized by $>3 \mathrm{eV}$, significantly more than typical solvent stabilization values. ${ }^{76,89,90}$ Furthermore, this step is not electrochemically driven, so even the application of large overpotentials will not enable direct N-N scission. This provides strong evidence against direct dissociation of the N-N bond on the pristine rutile $\mathrm{TiO}_{2}$ surface. The same stabilization would be required for $\mathrm{NH}^{*}$ species, effectively eliminating any pathway involving $\mathrm{NH}^{*}$ (e.g. dissociation of $\mathrm{NNH}$ ). Adsorbed $\mathrm{NH}_{2}$ species are somewhat more stable, and may exist under solvated conditions, opening the possibility of mechanisms involving dissociation of $\mathrm{N}_{2} \mathrm{H}_{\mathrm{x}>2}$ species, similar to the associative mechanism that will be discussed subsequently.

$$
\begin{aligned}
N_{2}(g)+^{*} & \rightarrow N_{2}^{*} \\
N_{2}^{*}+^{*} & \rightarrow 2 N^{*} \\
2 N^{*}+2\left(H^{+}+e^{-}\right) & \rightarrow 2 N H^{*} \\
2 N H^{*}+2\left(H^{+}+e^{-}\right) & \rightarrow 2 N H_{2}^{*} \\
2 N H_{2}^{*}+2\left(H^{+}+e^{-}\right) & \rightarrow 2 N H_{3}^{*} \\
N H_{3}^{*} & \rightarrow N H_{3}(g)+^{*}
\end{aligned}
$$

The associative nitrogen reduction mechanism proceeds via diazene $\left(\mathrm{N}_{2} \mathrm{H}_{2}\right)$ and hydrazine $\left(\mathrm{N}_{2} \mathrm{H}_{4}\right)$, as shown in equations 7 - 14. This mechanism has been proposed to be most relevant for electrochemical nitrogen reduction, ${ }^{25,74,91}$ and the fact that hydrazine has been observed as a photoreduction product on $\mathrm{TiO}_{2}{ }^{7}$ suggests that it may be the relevant photocatalytic 


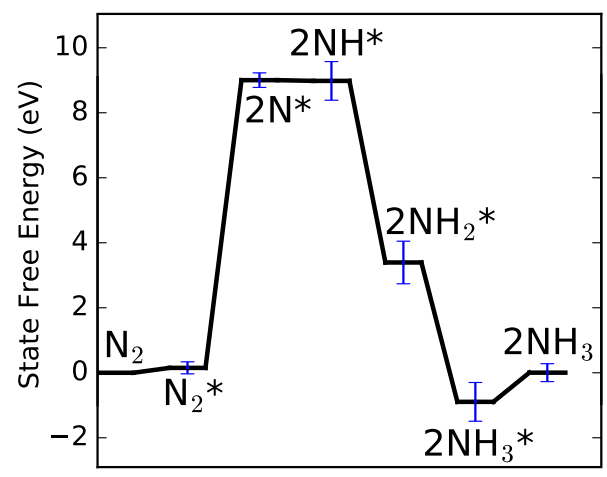

Reaction Coordinate

Figure 4: Free energy diagram for dissociative nitrogen reduction at the equilibrium potential computed from DFT (0.008 V, compared to $0.05 \mathrm{~V}$ from experiment). The blue error bars represent one standard deviation of the BEEF-vdW energy ensemble. Adsorbed states are labeled, and the full reaction mechanism is listed in equations 1 - 6 .

nitrogen reduction mechanism. The free energy diagram for the associative mechanism at the equilibrium potential is shown in Fig. 5a, while the free energy diagram at the conduction band edge energy for rutile is shown in Fig. 5b. Under photocatalytic conditions the conduction band edge is the relevant potential, corresponding to an overpotential of $0.15 \mathrm{~V}$. However, examination of the free energy diagram reveals a thermodynamic limiting potential of $2.5 \mathrm{~V}$ due to the unstable $\mathrm{NNH}^{*}$ adsorbed intermediate, which is consistent with previous work. ${ }^{25}$ This barrier is significantly higher than the conduction band potential, making the route improbable unless the adsorbates are stabilized significantly $(\approx 2 \mathrm{eV})$ by solvent/dipole effects. The rate-limiting hydrogenation of $\mathrm{N}_{2}$ is consistent with studies of electrochemical nitrogen reduction on metals, ${ }^{74}$ nitrides, ${ }^{92}$ and oxides ${ }^{25}$ indicating a general trend for photo- and electrochemical nitrogen reduction. 


$$
\begin{gathered}
N_{2}(g)+^{*} \rightarrow N_{2}^{*} \\
N_{2}^{*}+H^{+}+e^{-} \rightarrow N N H^{*} \\
N N H^{*}+H^{+}+e^{-} \rightarrow H N N H^{*} \\
H N N H^{*}+H^{+}+e^{-} \rightarrow H N N H_{2}^{*} \\
H N N H_{2}^{*}+H^{+}+e^{-} \rightarrow H_{2} N N H_{2}^{*} \\
H_{2} N N H_{2}^{*}+^{*}+H^{+}+e^{-} \rightarrow N H_{2}^{*}+N H_{3}^{*} \\
N H_{2}^{*}+N H_{3}^{*}+H^{+}+e^{-} \rightarrow 2 N H_{3}^{*} \\
2 N H_{3}^{*} \rightarrow 2 N H_{3}(g)+2^{*}
\end{gathered}
$$

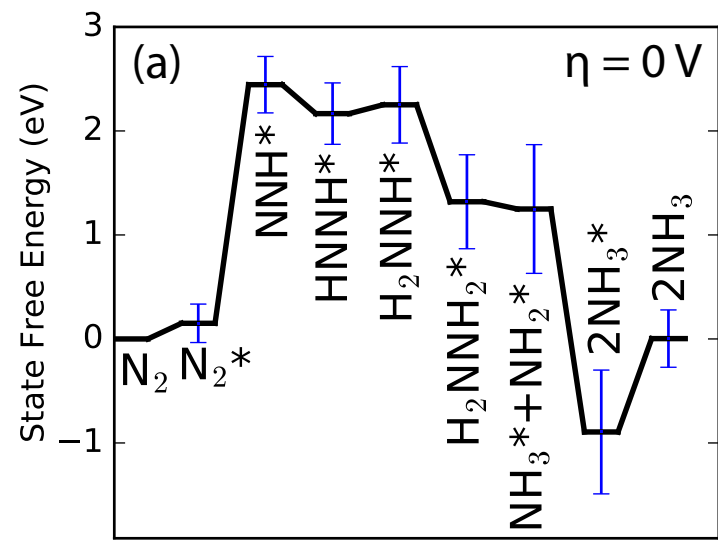

Reaction Coordinate

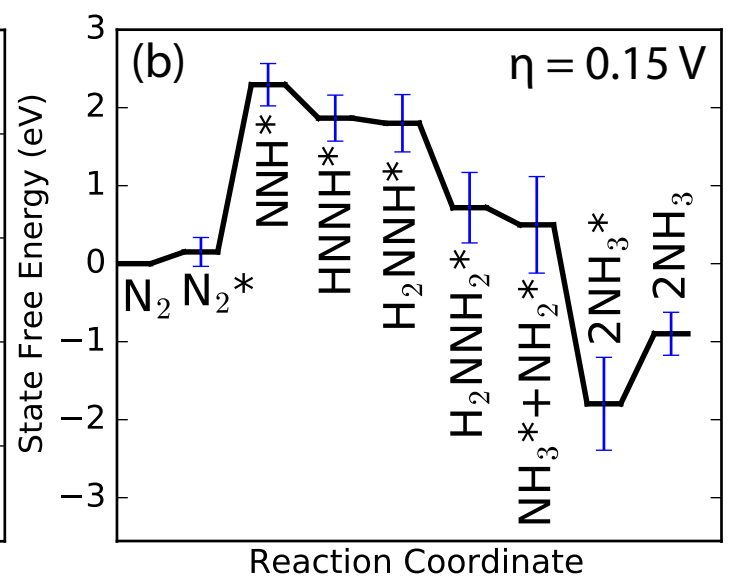

Figure 5: Free energy diagram for associative nitrogen reduction at an overpotential $(\eta)$ of zero (a) and at the overpotential due to the conduction band edge $(\eta=0.15 \mathrm{~V})(\mathrm{b})$. The equilibrium potential $(\eta=0)$ is computed to be $0.008 \mathrm{~V}(0.05 \mathrm{~V}$ experimentally). The blue error bars represent one standard deviation of the BEEF-vdW energy ensemble. Adsorbed states are labeled, and the full reaction mechanism is listed in equations 7 - 14 .

The results indicate a prohibitively high barrier for both dissociative and associative nitrogen reduction on pristine rutile $\mathrm{TiO}_{2}$ (110), although the associative pathway is significantly more favorable than the dissociative pathway. These findings are robust to the error of the exchange-correlation approximation employed, and the energetic barriers are signifi- 
cantly larger than the typical magnitude of electrochemical interface effects that have been neglected (solvent, electric field). This evidence refutes the hypothesis that rutile (110) is the active site for photocatalytic nitrogen reduction on $\mathrm{TiO}_{2}$, necessitating the development and testing of alternative hypotheses.

\section{Nitrogen reduction at oxygen vacancies and iron substitutions}

Surface defects are known to play a key role in many types of heterogeneous catalysis, ${ }^{93}$ and oxygen vacancies in particular have been shown to participate in numerous catalytic reactions on oxides ${ }^{94}$ including $\mathrm{TiO}_{2} \cdot{ }^{33,34,53,54,67}$ Oxygen vacancies are typically highly reactive, leading to binding energies that are often substantially stronger than binding at the stoichiometric surface. The unstable nature of $\mathrm{N}_{2} \mathrm{H}$ on pristine rutile (110) (Fig. 5) indicates that defect sites may enable nitrogen reduction by enhancing the stability of $\mathrm{N}_{2} \mathrm{H}$ and other high-energy intermediates. Bridging oxygen (O-br) vacancies are known to occur commonly on rutile (110) surfaces, ${ }^{53,54,67}$ and a recent analysis of nitrogen reduction over titania has shown that the reaction rate is proportional to the measured number of oxygen defects. ${ }^{9}$ Due to these considerations the O-br vacancy is a natural starting point for evaluating the effect of surface defects, although it is noted that other intrinsic defects, such as Ti vacancies in the surface or sub-surface may also play a role. ${ }^{95}$

The energetics of the associative nitrogen reduction pathway at the rutile (110) O-br defect site are shown in Fig. 6. Comparison to the energetics of the pristine surface (Fig. 8a) reveals a significant stabilization of the $\mathrm{N}_{2} \mathrm{H}$ intermediate, corresponding to a thermodynamic limiting potential of $1.21 \mathrm{~V}$. However, examination of the energy diagram at the conduction band potential of rutile $\mathrm{TiO}_{2}$ (Fig. 6b) indicates that the $\mathrm{N}_{2} \mathrm{H}$ and $\mathrm{N}_{2} \mathrm{H}_{2}$ intermediates are still too unstable to explain the photocatalytic reduction observed, although it is plausible that the thermodynamic barrier of $1.39 \mathrm{eV}$ (energy of $\mathrm{N}_{2} \mathrm{H}_{2}$ at the conduction band edge) may be overcome by stabilization if solvent or dipole effects for $\mathrm{N}_{2} \mathrm{H}$ and $\mathrm{N}_{2} \mathrm{H}_{2}$ are considerably larger than typical amounts of $\approx 0.6 \mathrm{eV} .{ }^{76,89,90}$ Furthermore, other defects 

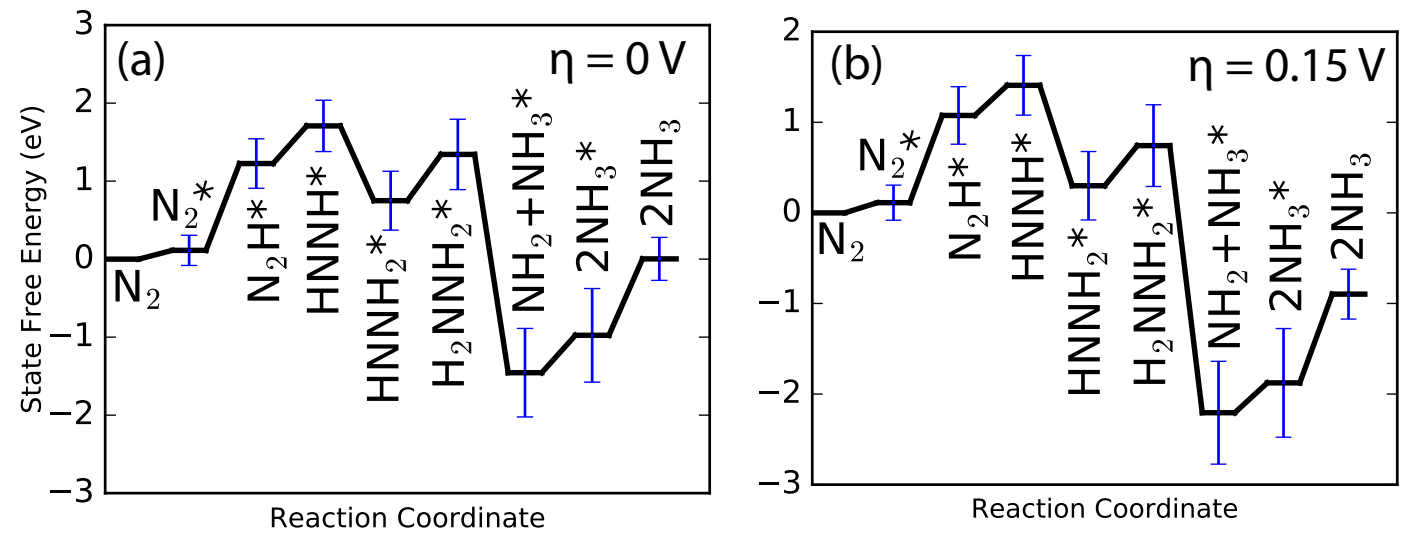

Figure 6: Free energy diagram for associative nitrogen reduction at an O-br vacancy site at an overpotential $(\eta)$ of zero (a) and at the overpotential due to the conduction band edge $(\eta=0.15 \mathrm{~V})(\mathrm{b})$. The equilibrium potential $(\eta=0)$ is computed to be $0.008 \mathrm{~V}(0.05 \mathrm{~V}$ experimentally). The blue error bars represent one standard deviation of the BEEF-vdW energy ensemble. Adsorbed states are labeled, and the full reaction mechanism is listed in the Supporting Information.

such as sub-surface O or Ti vacancies may increase the reactivity of the surface, although as defects become less stable they will also become less prevalent on the surface. This tradeoff between stability and reactivity has been noted previously for amorphous oxides, ${ }^{96}$ and suggests that more reactive (unstable) defects will have a limited impact due to low prevalence. The O-br defect considered here has a formation free energy of $1.54 \mathrm{eV}$ at $0 \mathrm{~V}$ SHE referenced against water, indicating that it will occur with an relatively low probability on thermodynamically equilibrated surfaces. Morphological (e.g. particle edges) and kinetic (e.g. trapped bulk defects) effects will increase the prevalence of vacancies or other defects in real catalysts; ${ }^{66}$ these effects are difficult to control and characterize, and may be the source of some discrepancies in the nitrogen photofixation literature. ${ }^{10}$

In addition, many experimental studies have noted that iron dopants significantly increase the nitrogen photofixation rates. ${ }^{2,3,5,6}$ Iron dopants may affect catalyst activity through effects in the bulk or on the surface chemistry. In the bulk, iron has been shown to improve charge separation, reducing recombination of electron-hole pairs. This charge separation enhancement has been suggested to be the dominant effect of iron dopants for photocatalytic 

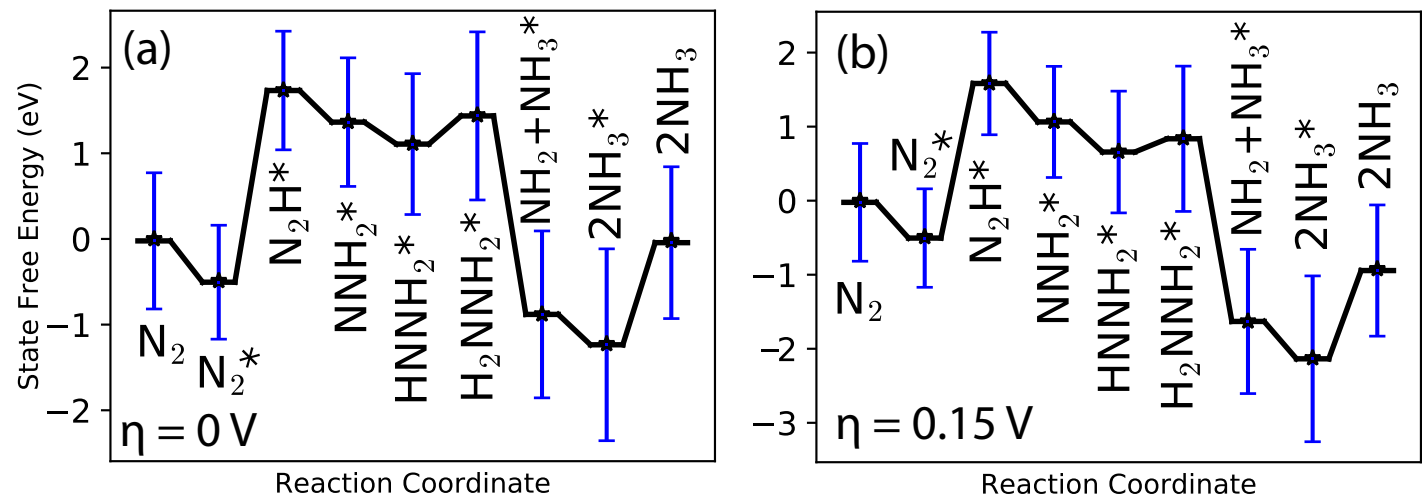

Figure 7: Free energy diagram for associative nitrogen reduction at an iron substitution site at an overpotential $(\eta)$ of zero (a) and at the overpotential due to the conduction band edge $(\eta=0.15 \mathrm{~V})(\mathrm{b})$. The equilibrium potential $(\eta=0)$ is computed to be $0.008 \mathrm{~V}(0.05 \mathrm{~V}$ experimentally). The blue error bars represent one standard deviation of the BEEF-vdW energy ensemble. Adsorbed states are labeled, and the full reaction mechanism is listed in the Supporting Information.

nitrogen fixation on titania. ${ }^{6}$ Alternatively, an iron substitution at the surface may stabilize the states along the reductive pathway, directly improving the energetics of the process. The latter hypothesis was tested by computing the energetics of the associative mechanism on a slab with an iron substitution defect. Two defects were considered, an $\mathrm{Fe}^{4+}$ defect arising from direct substitution of the 5-fold $\mathrm{Ti}$ atom, and an $\mathrm{Fe}^{2+}$ defect formed by substitution of a Ti atom beneath a bridging $\mathrm{O}$ and removal of the bridging $\mathrm{O}$, effectively forming a O-br defect and Fe substitution (see the Supplementary Information for visualization of the slab). The $\mathrm{Fe}^{2+}$ defect was found to be more stable, and Fig. 7 shows the energetics of the associative pathway with an iron-substituted rutile (110) surface (a comparison of the free energy path for the $\mathrm{Fe}^{4+}$ defect is available in the Supplementary Information). This mechanism is very similar to the mechanism on the pristine and O-br vacancies, with the slight difference

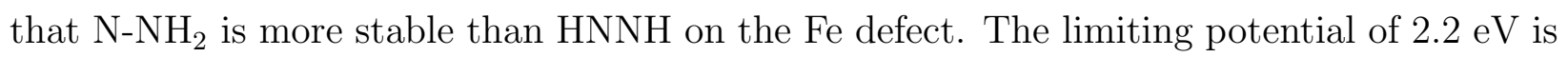
comparable to that of the defected surface in Fig. $6(1.7 \mathrm{eV})$, but is slightly higher due to the stronger adsorption of $\mathrm{N}_{2}$. The energetics of the associative pathway on Fe-substitution defects are compared directly to O-br defects and pristine rutile (110) in Fig. 8a, illustrating 
that the Fe-substitution defect has a similar effect to the O-br vacancy. The energy required to form this defect was calculated to be $1.1 \mathrm{eV}$ relative to bulk rutile and BCC iron. This moderate formation energy is lower than that of the O-br defect, and $\mathrm{N}_{2}$ adsorbs with a relatively strong binding energy of $-0.5 \mathrm{eV}$. This suggests that Fe surface defects promote the formation of O-br vacancies and adsorption of $\mathrm{N}_{2}$. Nonetheless, the high limiting potential of $2.2 \mathrm{~V}$ indicates that the Fe-substitution defect is not active for photocatalytic nitrogen reduction.

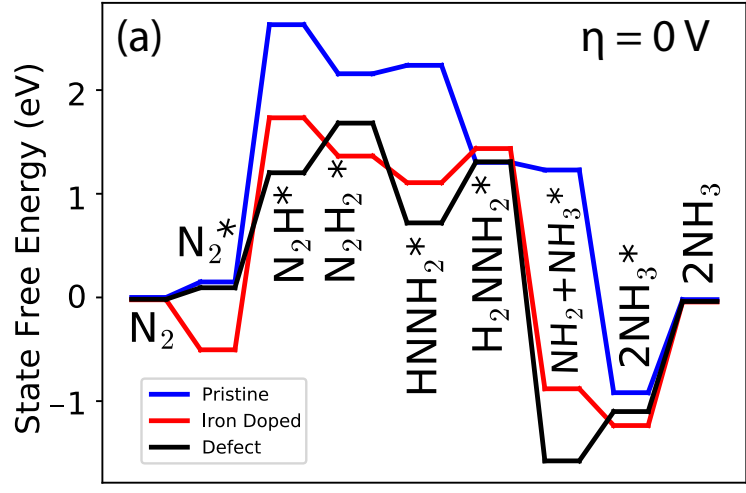

Reaction Coordinate

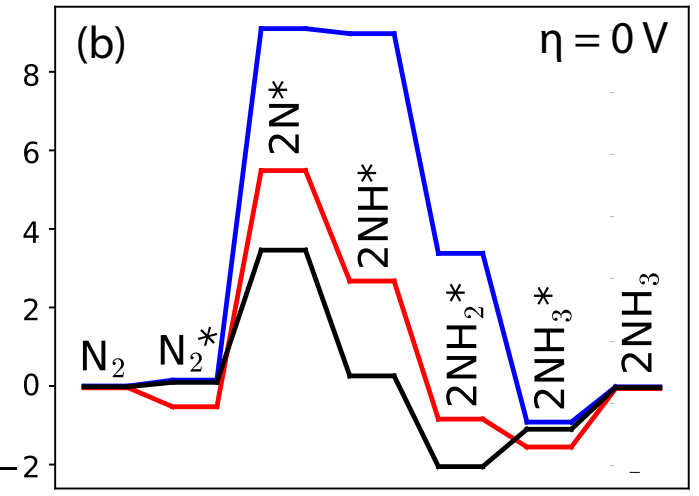

Reaction Coordinate

Figure 8: Comparison of free energy pathways over pristine (blue), iron-doped (red) and oxygen-vacancy defect (black) for the associative (a) and dissociative (b) nitrogen reduction pathways at the equilibrium potential. All details are consistent with Figs. 4a - 7a. Error bars are omitted for clarity.

Another possibility is that O-br vacancies and/or Fe-substitution defects significantly impact the energetics of the dissociative pathway. Iron catalysts are known to activate the $\mathrm{N}-\mathrm{N}$ bond in the Haber-Bosch process,${ }^{84,87}$ suggesting that the Fe site may play a role, and Hirakawa et. al. ${ }^{9}$ have hypothesized that direct N-N bond scission at O-br vacancies is the mechanism for photocatalytic nitrogen fixation. The energetics of the dissociative pathway for both Fe-substitution and O-br vacancy defects are compared with the energetics of the pristine surface in Fig. 8b. The results show that the Fe-substitution has a relatively small effect on the thermodynamics of $\mathrm{N}-\mathrm{N}$ bond scission, although a more pronounced effect is seen for $\mathrm{NH}^{*}$ intermediates that are stabilized by $>1 \mathrm{eV}$. The $\mathrm{O}-\mathrm{br}$ vacancies have a much larger effect on $\mathrm{N}-\mathrm{N}$ bond scission, stabilizing adsorbed $\mathrm{N}^{*}$ by $>2 \mathrm{eV}$ per adsorbate. However, 
the thermodynamic barrier of $\approx 4 \mathrm{eV}$ is still prohibitive at ambient conditions, and significantly higher than the $1.21 \mathrm{~V}$ thermodynamic limiting potential needed for the associative mechanism at the O-br vacancy. An alternative possibility is a mixed mechanism proceeding through dissociaton of partially hydrogenated species, since the $\mathrm{NH}_{\mathrm{x}}$ species are stable at the O-br vacancy; however, this would still necessitate the formation of the potential-limiting $\mathrm{HNNH}^{*}$ species from the associative mechanism and would be thermodynamically (though not kinetically) equivalent. Furthermore, we note that the free energy diagram in Fig. 8b requires two O-br vacancies, since each $\mathrm{N}^{*}\left(\right.$ or $\left.\mathrm{NH}_{\mathrm{x}}\right)$ is adsorbed at a vacancy. The relatively high formation energy of O-br vacancies suggests that this is improbable, and the experimental investigation of Hirakawa et. al. ${ }^{9}$ shows a linear dependence on oxygen vacancies, rather than the quadratic dependence that would be indicative of direct N-N (or HN-NH) scission by two vacancy sites. This finding strongly refutes the proposed hypothesis that direct N-N scission by $\mathrm{O}-\mathrm{br}$ vacancies is the mechanism of photocatalytic nitrogen fixation on $\mathrm{TiO}_{2}$. However, the fact that the O-br vacancy significantly stabilizes $\mathrm{NH}_{\mathrm{x}}$ species, making $\mathrm{NH}_{\mathrm{x}}$ binding close to exothermic suggests that it can promote nitrogen reduction and ammonia formation after the N-N bond has been cleaved.

\section{Nitrogen oxidation and indirect reduction}

The majority of experimental investigations of photocatalytic nitrogen fixation on $\mathrm{TiO}_{2}$ catalysts have identified reduced products of ammonia or ammonium. However, several reports have observed nitrates as the main product, ${ }^{4,8}$ and examination of the standard redox potentials and $\mathrm{TiO}_{2}$ band edges (3) indicates that the band alignment for nitrogen oxidation to $\mathrm{NO}$ is significantly more favorable on $\mathrm{TiO}_{2}$ than the band alignment for nitrogen reduction. The band alignment provides $\approx 1.25 \mathrm{~V}$ overpotential for both the oxygen reduction half-reaction and the oxidation of nitrogen for NO. Based on this we hypothesize that the scission of the N-N bond on rutile (110) proceeds via the oxidation of nitrogen to NO, which is subsequently oxidized or reduced depending on the details of the catalyst and reaction 
conditions.

The thermodynamics of nitrogen oxidation intermediates on rutile (110) are shown in Fig. 9 at the equilibrium potential (a) and the energy of the $\mathrm{TiO}_{2}$ valence band edge (b). Examination of the free energy profile at the equilibrium potential (Fig. 9a) reveals that the thermodynamic limiting potential is $0.72 \mathrm{~V}$ (surface oxygen formation is the potentiallimiting step), considerably lower than the case of nitrogen reduction. In addition, the direct adsorption of $\mathrm{N}_{2}$ to this reactive surface oxygen is exergonic by $0.3 \mathrm{eV}$, indicating that adsorption of $\mathrm{N}_{2}$ is favored by oxygen-rich surfaces. When the significant driving force provided by the photo-excited hole (1.22 V overpotential) is taken into account (Fig. 9b) the oxidative path becomes extremely favorable, with all steps being exergonic with the exception of $\mathrm{N}_{2} \mathrm{O}_{2}$ dissociation which is very slightly $(<0.1 \mathrm{eV})$ uphill. We note that this analysis considers only the thermodynamics of adsorbed states, and neglects activation barriers that will ultimately govern the kinetics of the process. These barriers could potentially be significant for $\mathrm{N}-\mathrm{O}$ coupling, although the strong driving force under photocatalytic conditions will improve the kinetics of any electrochemical step.

(a)

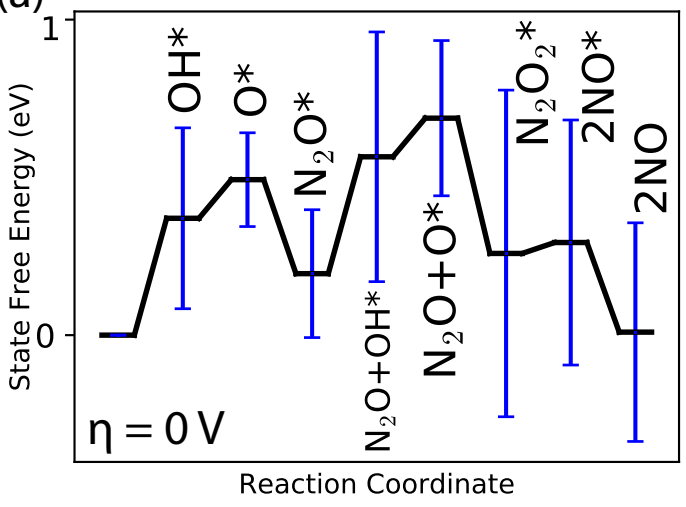

(b)

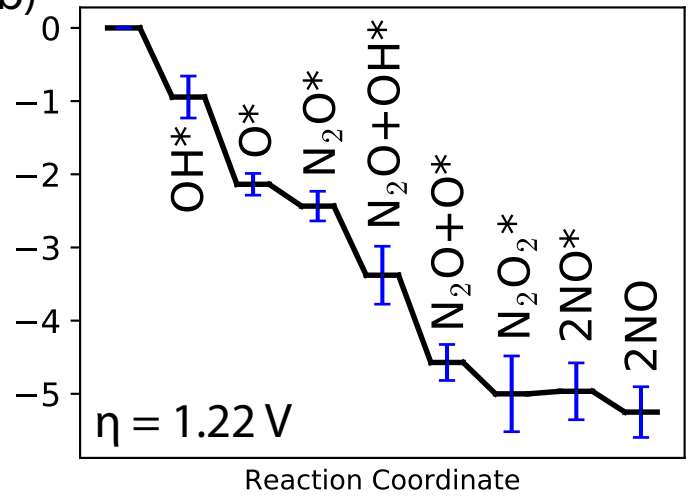

Figure 9: Free energy diagram for nitrogen oxidation to NO at an overpotential $(\eta)$ of zero (a) and at the overpotential due to the valence band edge $(\eta=1.22 \mathrm{~V})$ (b). The equilibrium potential $(\eta=0)$ is computed to be $1.465 \mathrm{~V}(1.68 \mathrm{~V}$ experimentally). The blue error bars represent one standard deviation of the BEEF-vdW energy ensemble. Adsorbed states are labeled, and the full reaction mechanism is listed in the Supporting Information.

The computational results provide strong evidence that nitrogen oxidation is thermody- 
namically feasible on the rutile (110) active site, while $\mathrm{N}_{2}$ reduction is thermodynamically challenging. This is at odds with the experimental observation of reduced products on $\mathrm{TiO}_{2} \cdot{ }^{2,3,5-7,9}$ One possible explanation is that nitrogen is first oxidized to NO and subsequently reduced to ammonia. The conversion of $\mathrm{NO}$ to $\mathrm{NH}_{3}$ is a 5 electron process with a redox potential at $0.71 \mathrm{~V}$ vs. $\mathrm{RHE}$, well below the band gap of $\mathrm{TiO}_{2},{ }^{10}$ and has been reported experimentally ${ }^{97}$ and studied theoretically. ${ }^{26}$ The thermodynamic feasibility of this pathway on rutile (110) has been computed and the most thermodynamically favorable path is shown in Fig. 10. The mechanism and energetics are consistent with prior work, ${ }^{26}$ and indicate that this is indeed thermodynamically feasible. However, reduction of nitrogen oxides is a complex process that can also form partially reduced species such as $\mathrm{N}_{2} \mathrm{O}$ or $\mathrm{N}_{2}$. In particular, the reaction of $\mathrm{NO}$ to $\mathrm{N}_{2} \mathrm{O}$ and the reaction of $\mathrm{N}_{2} \mathrm{O}$ to $\mathrm{N}_{2}$ has been observed under UHV conditions by Yates and colleagues. ${ }^{18,19}$ Fully understanding the selectivity of photocatalytic $\mathrm{NO}$ reduction on $\mathrm{TiO}_{2}$ is beyond the scope of this work, but selectivity should be considered in future studies of NO reduction.

Experimentally, titania photocatalysts have been reported to reduce nitrates under aqueous conditions, although selectivity to dinitrogen vs. ammonia varies widely based on preparation conditions and metal dopants. ${ }^{26,97-101}$ Contrarily, oxidation of ammonia has also been reported for $\mathrm{TiO}_{2}$ photocatalysts, ${ }^{102-104}$ as well as simultaneous reduction of nitrate and oxidation of ammonium to $\mathrm{N}_{2} \cdot{ }^{104}$ Furthermore, the observation of hydrazine as a product from $\mathrm{TiO}_{2}$ nitrogen photofixation is not explained by this mechanism. ${ }^{7}$ These conflicting results suggest that other hypotheses should also be considered. In particular, the formation of hydrazine should be examined more closely to determine if hydrazine could be formed through recombination of $\mathrm{NH}_{x}$ species (consistent with the oxidative pathway) or if it is formed associatively (consistent with the reductive pathway). Isotopic scrambling experiments ${ }^{105}$ and use of photoelectrochemical applied bias experiments may provide insight into this outstanding question. 

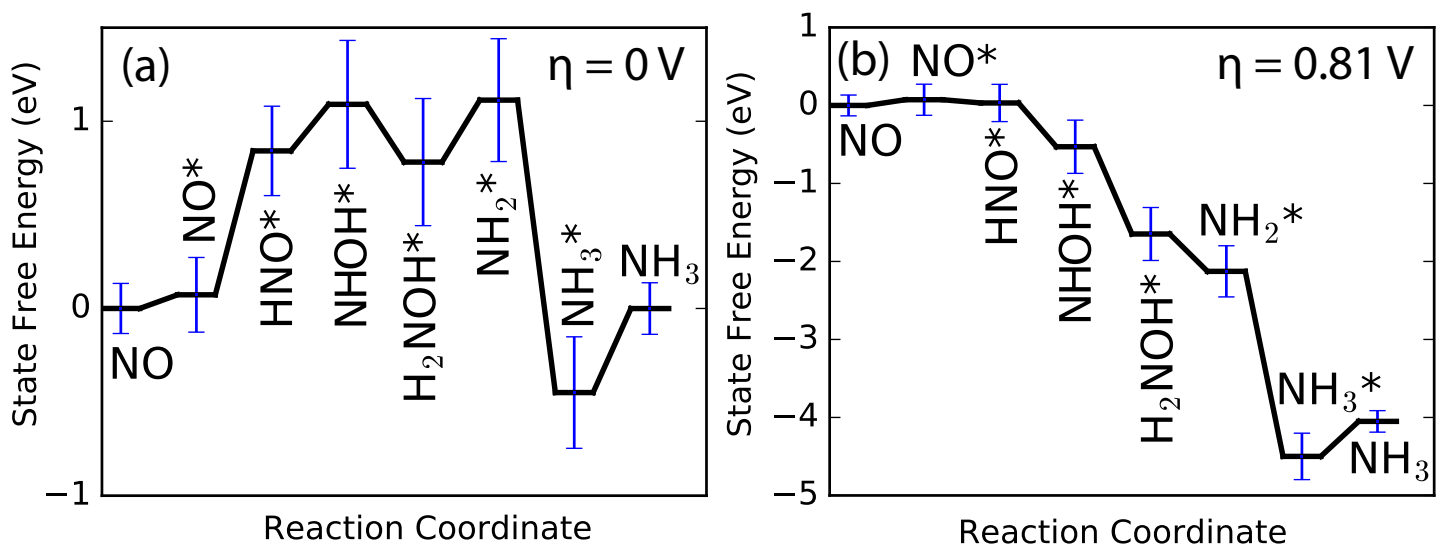

Figure 10: Free energy diagram for $\mathrm{NO}$ reduction to $\mathrm{NH}_{3}$ at an overpotential $(\eta$ ) of zero (a) and at the overpotential due to the valence band edge $(\eta=0.81 \mathrm{~V})(\mathrm{b})$. The equilibrium potential $(\eta=0)$ is computed to be $0.59 \mathrm{~V}(0.71 \mathrm{~V}$ experimentally). The blue error bars represent one standard deviation of the BEEF-vdW energy ensemble. Adsorbed states are labeled, and the full reaction mechanism is listed in the Supporting Information.

\section{Conclusions}

The hypothesis that any of the sites on pristine rutile (110) are the active site for nitrogen reduction was shown to be false based on the results of BEEF-vdW DFT calculations for both the associative and dissociative mechanism, although the associative mechanism was found to be considerably more thermodynamically favorable. The revised hypothesis that oxygen vacancies or Fe substitutions are active sites was shown to be more plausible, and a significant stabilization of $\mathrm{NH}_{\mathrm{x}}$ by O-br vacancies was found. Yet, the predicted thermodynamic barriers are considerably higher than the conduction band edge, and the defects are not predicted to be thermodynamically stable under operating conditions, leading to the conclusion that this hypothesis is also improbable. However, the results indicate that $\mathrm{N}-\mathrm{N}$ bond cleavage is thermodynamically facile on rutile (110) through an oxidative pathway, particularly with the strong oxidative driving force provided by photogenerated holes. Based on this finding, the hypothesis of an oxidized $\mathrm{NO}^{*}$ intermediate in nitrogen reduction is introduced as a possible mechanism for photocatalytic nitrogen fixation on rutile (110). The work provides initial molecular-scale insight into the mechanisms that underly photocatalytic nitrogen fixation 
on $\mathrm{TiO}_{2}$ by conclusively eliminating several possible explanations for this important process and identifying a novel hypothesis of indirect reduction through an oxidized intermediate.

\section{Methods}

\section{Computational}

\section{Density Functional Theory}

In the current work the Quantum ESPRESSO software package ${ }^{106}$ is used in conjunction with the Atomic Simulation Package (ASE) ${ }^{107}$ to carry out plane wave density functional theory calculations. The BEEF-vdw functional ${ }^{75}$ is used for all work with a plane wave cuttoff of $400 \mathrm{eV}$ and a 4x4x1 Monkhorst-Pack k-point grid is used for all slab models. ${ }^{108}$ Spin polarization and a dipole correction ${ }^{109}$ (applied along the axis of the slab) are used for all slab calculations. Structures are optimized using the BFGS line search method to a total force of $0.05 \mathrm{eV} / \AA$. The geometries and adsorption energies for all species are available in Tables S1 and S3. All gas-phase species are evaluated in a periodic unit cell with $6 \AA$ of vacuum space and a $\Gamma$-point k-point sampling. A number of calculated properties of $\mathrm{TiO}_{2}$ (e.g. band gap, water adsorption energy) vary with the number of layers in the model slab; ${ }^{50,110,111}$ slabs with an even number of layers are known to be more accurate due to a symmetric electronic environment between sets of layers. ${ }^{50}$ The error due to slab size is expected to be ca. $0.2 \mathrm{eV},{ }^{50}$ similar to the error of DFT for adsorption energies. ${ }^{112}$ Furthermore, there is debate regarding the accuracy of GGA functionals for $\mathrm{TiO}_{2}$. Several studies have indicated that catalytic

properties, even of defects, can be reliably treated with GGA functionals, ${ }^{57,113,114}$ although other work indicates that hybrid, $+\mathrm{U}$, or double-hybrid methods are necessary, particularly to treat electronic transitions and optical properties. ${ }^{115-118}$ In this work we utilize the error estimation capabilities of the BEEF-vdW functional ${ }^{75}$ in order to estimate its accuracy and quantify the uncertainty due to the GGA approximation. Although the quantitative 
accuracy of BEEF-vdW error estimation in oxide materials is not well-known, ${ }^{119}$ the BEEFvdW ensembles provide a systematic way to assess the sensitivity of conclusions to accuracy of approximations. ${ }^{120}$ The error bars shown in this work correspond to $\pm 1 \sigma$ of the ensemble of 2000 energies provided by the BEEF-vdW functional. The uncertainty quantified in the BEEF ensembles is also propagated to phase diagrams (Fig. 2). The probability of a species on the surface is defined as:

$$
P_{i}=\frac{1}{N} \sum_{l}^{N} \frac{\exp \left(\frac{-G_{i}^{l}}{k T}\right)}{\sum_{j}^{M} \exp \left(\frac{-G_{j}^{l}}{k T}\right)}
$$

where $P_{i}$ is the probability of species $i$ given DFT uncertainty, $G_{i}^{l}$ is the free energy of species $i$ computed from energy $l$ of the BEEF-vdW ensemble, $M$ are the number of total species considered, and $N$ is the total number of energies in the BEEF-vdW ensemble (2000). This is equivalent to the average surface coverage from a ensemble of phase diagrams generated from the energies of the BEEF-vdW ensemble.

\section{Thermochemistry}

Ground state electronic energies $\left(E_{\text {ele }}\right)$ are obtained from DFT and converted to free energies by including zero point energy (ZPE) and thermal contributions:

$$
G_{i}^{o}=E_{e l e}+E_{Z P E}+\Delta H-T \Delta S
$$

where $E_{Z P E}$ is the zero-point energy and $\Delta H$ (enthalpy) and $\Delta S$ (entropy) are thermal contributions computed using the vibrational frequencies computed through a finite difference approximation to the Hessian. Adsorbates are treated using the harmonic approximation with a low-frequency cutoff of $30 \mathrm{~cm}^{-1}$, while gas phase molecules were treated as ideal gases. The ASE implementation ${ }^{107}$ is used for vibrational analysis and statistical mechanics corrections. Vibrational frequencies for all species are reported in Table S2. Vibrational frequencies of adsorbates at defect sites are assumed to be the same as vibrational frequencies 
on the pristine surface. All thermodynamics were evaluated at $300 \mathrm{~K}$ and gas partial pressures were set approximate atmospheric conditions ( 0.8 atm $\mathrm{N}_{2}, 0.2 \operatorname{atm~} \mathrm{O}_{2}$ ). Liquid water was approximated as an ideal gas at saturation pressure at $300 \mathrm{~K}$ (0.035 atm). Relative (formation) energies were computed with respect to reference states using the equation:

$$
G_{i}=G_{i}^{o}-\sum_{j} n_{j} \mu_{j}
$$

where $G_{i}$ is the formation free energy of species $i, G_{i}^{o}$ is the "absolute" free energy computed from DFT and free energy corrections (see Equation 16), $n_{i}$ is the number of atoms $j$ in species $i$, and $\mu_{i}$ is the reference chemical potential. The reference for nitrogen is $\mathrm{N}_{2}\left(\mu_{N}=\right.$ $\left.\frac{1}{2} G_{N_{2}}^{o}\right)$, the reference for oxygen is $\mathrm{H}_{2} \mathrm{O}\left(\mu_{H_{2} O}=\frac{1}{2} G_{H_{2} O}^{o}-\mu_{H}\right)$ and the reference for hydrogen is set by the computational hydrogen electrode $\left(\mu_{H}=\frac{1}{2} G_{H_{2}}^{o}+\mathrm{eU}\right)$. In this work no gas-

phase corrections (e.g. $\mathrm{O}_{2},{ }^{71} \mathrm{CO}_{2}{ }^{73}$ ) are applied since it is difficult to rigorously correct the BEEF-vdW ensembles; the quantification and propagation of error with the BEEF-vdW ensembles will capture these errors to some extent, and overpotentials are defined relative to the equilibrium potential computed by DFT in order to minimize the influence of inaccurate equilibrium potentials from DFT (see following section).

\section{Photoelectrochemistry}

The photoelectrochemical model used assumes that excitation, charge transport, and charge transfer are decoupled from electrochemistry and is largely consistent with the approach reviewed by Hellman and Wang. ${ }^{76}$ All states involving electrons utilized the CHE model reaction as a reference electrode. This model sets the free energy of reaction of hydrogen splitting, reaction 18, to zero at a potential of zero. This "computational hydrogen electrode" (CHE) is conceptually equivalent to the reversible hydrogen electrode (RHE). ${ }^{73}$

The energy of each state involving the addition of an electron is varied by an energy of $e U$, where e is the fundamental change and $\mathrm{U}$ is the potential relative to this zero point, at 
potentials other than zero reaction:

$$
\frac{1}{2} H_{2} \rightarrow H^{+}+e^{-}
$$

This model was also used to assess the effect of photo-excited electrons and holes. The locations of the band edges were obtained from the literature ${ }^{81}$ and used to set the potentials of the electrons and holes relative to a the redox couples of the relevant reactions so that the overpotential is equivalent to the experimentally expected overpotential. This approach causes the absolute potential of electrons/holes to depend on the reaction in question (due to DFT errors in the reaction energies), while the relative energy of electrons/holes to a given redox couple is equal to the experimental value. The computed (and experimental) equilibrium potential of a given reaction is provided in the caption of each figure.

\section{Determination of adsorption sites}

Several adsorption sites were tested for stability for all stable molecular species and adatoms. For most stable molecular species the only viable adsorption site was the 5 -fold titanium site; attempts to adsorb species to the bridging oxygen or in plane oxygens either led to desorption or reorientation to the 5-fold titanium site. These findings are in agreement with previous first-principles examinations. ${ }^{21,22}$ Adatoms $\left(\mathrm{N}^{*}, \mathrm{O}^{*}, \mathrm{H}^{*}\right)$ were found to have multiple adsorption sites. Oxygen was found to adsorb to the 5-fold titanium site or form a diatomic species at the bridging oxygen site. The latter can be idenified as the $\alpha$-oxygen species reported by Lu. ${ }^{17}$ Hydrogen adatoms were found to be most stable at the in plane oxygen, and the nitrogen adatom was found to have highest stability over the bridging oxygen. A full list of energies and structures is provided in the Supplementary Information. 


\section{Acknowledgement}

The authors thank the School of Chemical \& Biomolecular Engineering at the Georgia Institute of Technology for providing start-up funding for this work. The authors are also grateful to Marta C. Hatzell for constructive discussions, and Timothy Doane for providing references regarding photocatalytic nitrogen fixation.

\section{Publication Disclosure}

This document is the unedited Author's version of a Submitted Work that has been submitted for publication in ACS Sustainable Chemistry and Engineering, copyright American Chemical Society.

\section{Supporting Information Available}

The following files are available free of charge. The Supporting Information is available and contains:

All adsorption energies, vibrational frequencies, and structure images; surface free energy, coverage, and probability diagrams including all species under oxidizing and reducing conditions; reaction equations for all mechanisms and defect formations; comparison of free energy pathway for different Fe substitution models.

\section{References}

1. Dhar, N.; Seshacharyulu, E.; Biswas, N. New aspects of nitrogen fixation and loss in soils. Proc. Natl. Inst. Sci. India 1941, 7, 115-131.

2. Schrauzer, G.; Guth, T. Photocatalytic reactions. 1. Photolysis of water and photoreduction of nitrogen on titanium dioxide. J. Am. Chem. Soc. 1977, 99, 7189-7193. 
3. Schrauzer, G. N.; Strampach, N.; Hui, L. N.; Palmer, M. R.; Salehi, J. Nitrogen photoreduction on desert sands under sterile conditions. Proc. Natl. Acad. Sci. U.S.A. 1983, $80,3873-3876$.

4. Bickley, R. I.; Vishwanathan, V. Photocatalytically induced fixation of molecular nitrogen by near UV radiation. Nature 1979, 280, 306-308.

5. Augugliaro, V.; Lauricella, A.; Rizzuti, L.; Schiavello, M.; Sclafani, A. Conversion of solar energy to chemical energy by photoassisted processes-I. Preliminary results on ammonia production over doped titanium dioxide catalysts in a fluidized bed reactor. Int. J. Hydrog. Energy 1982, 7, 845-849.

6. Soria, J.; Conesa, J. C.; Augugliaro, V.; Palmisano, L.; Schiavello, M.; Sclafani, A. Dinitrogen photoreduction to ammonia over titanium dioxide powders doped with ferric ions. J. Phys. Chem. 1991, 95, 274-282.

7. Schrauzer, G. N. Energy Efficiency and Renewable Energy Through Nanotechnology; Springer, 2011; pp 601-623.

8. Yuan, S.-J.; Chen, J.-J.; Lin, Z.-Q.; Li, W.-W.; Sheng, G.-P.; Yu, H.-Q. Nitrate formation from atmospheric nitrogen and oxygen photocatalysed by nano-sized titanium dioxide. Nat. Commun. 2013, 4 .

9. Hirakawa, H.; Hashimoto, M.; Shiraishi, Y.; Hirai, T. Photocatalytic Conversion of Nitrogen to Ammonia with Water on Surface Oxygen Vacancies of Titanium Dioxide. J. Am. Chem. Soc. 2017, 139, 10929-10936.

10. Medford, A. J.; Hatzell, M. C. Photon-Driven Nitrogen Fixation: Current Progress, Thermodynamic Considerations, and Future Outlook. ACS Catalysis 2017, 2624-2643.

11. Edwards, J. G.; Davies, J. A.; Boucher, D. L.; Mennad, A. An Opinion on the Heterogeneous Photoreactions of N2 with H2O. Angew. Chem. Int. Ed. 1992, 31, 480-482. 
12. Davies, J. A.; Edwards, J. G. Reply: Standards of Demonstration for the Heterogeneous Photoreactions of N2 with H2O. Angew. Chem. Int. Ed. 1993, 32, 552-553.

13. Boucher, D. L.; Davies, J. A.; Edwards, J. G.; Mennad, A. An investigation of the putative photosynthesis of ammonia on iron-doped titania and other metal oxides. Journal of J. Photochem. Photobiol., Abiology A: Chemistry 1995, 88, 53-64.

14. Davies, J. A.; Boucher, D. L.; Edwards, J. G. Adv. Photochem.; John Wiley \& Sons, Inc., pp 235-310.

15. Benkoula, S.; Sublemontier, O.; Patanen, M.; Nicolas, C.; Sirotti, F.; Naitabdi, A.; Gaie-Levrel, F.; Antonsson, E.; Aureau, D.; Ouf, F.-X.; Wada, S.-I.; Etcheberry, A.; Ueda, K.; Miron, C. Water adsorption on TiO2 surfaces probed by soft X-ray spectroscopies: bulk materials vs. isolated nanoparticles. Sci. Rep. 2015, 5, 15088.

16. Walle, L. E.; Borg, A.; Uvdal, P.; Sandell, A. Experimental evidence for mixed dissociative and molecular adsorption of water on a rutile $\mathrm{TiO} 2$ (110) surface without oxygen vacancies. Phys. Rev. B 2009, 80, 235436.

17. Lu, G.; Linsebigler, a.; Yates, J. T. Ti3+ Defect Sites on TiO2(110): Production and Chemical Detection of Active Sites. J. Phys. Chem. 1994, 98, 11733-11738.

18. Rusu, C. N.; Yates, J. T. Photochemistry of NO Chemisorbed on TiO $2(110)$ and TiO 2 Powders. J. Phys. Chem. B 2000, 104, 1729-1737.

19. Rusu, C. N.; Yates Jr., J. T. N2O Adsorption and Photochemistry on High Area TiO2 Powder. J. Phys. Chem. B 2001, 105, 2596-2603.

20. Henderson, M. A. A surface science perspective on TiO2 photocatalysis. Surf. Sci. Rep. 2011, 66, 185-297. 
21. Stodt, D.; Noei, H.; Hättig, C.; Wang, Y. A combined experimental and computational study on the adsorption and reactions of NO on rutile TiO2. Physical chemistry chemical physics : PCCP 2013, 15, 466-72.

22. Sorescu, D.; Rusu, C.; Jr, J. Y. Adsorption of NO on the TiO2 (110) Surface: An Experimental and Theoretical Study. J. Phys. Chem. B 2000, 2, 4408-4417.

23. Cheng, D.; Lan, J.; Cao, D.; Wang, W. Adsorption and dissociation of ammonia on clean and metal-covered TiO2 rutile (110) surfaces: A comparative DFT study. Appl. Catal., B 2011, 106, 510-519.

24. Diebold, U. The surface science of titanium dioxide. Surf. Sci. Rep. 2003, 48, 53-229.

25. Hskuldsson, Á. B.; Abghoui, Y.; Gunnarsdóttir, A. B.; Skúlason, E. Computational Screening of Rutile Oxides for Electrochemical Ammonia Formation. ACS Sustainable Chemistry \& Engineering 2017, 5, 10327-10333.

26. Xie, X.-Y.; Wang, Q.; Fang, W.-H.; Cui, G. DFT Study on Reaction Mechanism of Nitric Oxide to Ammonia and Water on a Hydroxylated Rutile TiO2(110) Surface. The J. Phys. Chem. C 2017, 121, 16373-16380.

27. Onal, I.; Soyer, S.; Senkan, S. Adsorption of water and ammonia on TiO2-anatase cluster models. Surf. Sci. 2006, 600, 2457-2469.

28. Erdogan, R.; Ozbek, O.; Onal, I. A periodic DFT study of water and ammonia adsorption on anatase TiO2 (001) slab. Surf. Sci. 2010, 604, 1029-1033.

29. Erdogan, R.; Onal, I. An ONIOM and DFT study of water and ammonia adsorption on anatase TiO2 (001) cluster. Int. J. Quantum Chem. 2011, 111, 2149-2159.

30. Markovits, A.; Ahdjoudj, J.; Minot, C. A theoretical analysis of NH3 adsorption on TiO2. Surf. Sci. 1996, 365, 649-661. 
31. Ji, Y.; Luo, Y. First-Principles Study on the Mechanism of Photoselective Catalytic Reduction of NO by NH3 on Anatase TiO2(101) Surface. J. Phys. Chem. C 2014, 118, 6359-6364.

32. jing Guo, X.; Liu, W.; Fang, W.; Cai, L.; Zhu, Y.; Lu, L.; Lu, X. DFT study of coverage-depended adsorption of NH3 on TiO2-B (100) surface. Phys. Chem. Chem. Phys. 2012, 14, 16618.

33. Kim, B.; Dohnálek, Z.; Szanyi, J.; Kay, B. D.; Kim, Y. K. Temperature-programmed desorption study of NO reactions on rutile TiO2(110)-1x1. Surf. Sci. 2016, 652, 148155.

34. Kim, B.; Li, Z.; Kay, B. D.; Dohnálek, Z.; Kim, Y. K. Low-Temperature Desorption of N 2 O from NO on Rutile TiO 2 (110)-1 x 1. The J. Phys. Chem. C 2014, 118, $9544-9550$.

35. Chen, H.; Nambu, A.; Wen,; Graciani, J.; Zhong,; Hanson, J. C.; Fujita, E.; Rodriguez, J. A. Reaction of NH3with Titania: N-Doping of the Oxide and TiN Formation. J. Phys. Chem. C 2007, 111, 1366-1372.

36. Karunagaran, B.; Uthirakumar, P.; Chung, S.; Velumani, S.; Suh, E.-K. TiO2 thin film gas sensor for monitoring ammonia. Mater. Charact. 2007, 58, 680-684.

37. Suganuma, S.; Murakami, Y.; Ohyama, J.; Torikai, T.; Okumura, K.; Katada, N. Assignments of Bending Vibrations of Ammonia Adsorbed on Surfaces of Metal Oxides. Catal. Lett. 2015, 145, 1904-1912.

38. Busca, G. Chemical and mechanistic aspects of the selective catalytic reduction of NOx by ammonia over oxide catalysts: A review. Appl. Catal., B 1998, 18, 1-36.

39. Ramis, G.; Busca, G.; Lorenzelli, V.; Forzatti, P. Fourier transform infrared study of 
the adsorption and coadsorption of nitric oxide, nitrogen dioxide and ammonia on TiO2 anatase. App. Catal. 1990, 64, 243-257.

40. Giraud, F.; Geantet, C.; Guilhaume, N.; Gros, S.; Porcheron, L.; Kanniche, M.; Bianchi, D. Experimental Microkinetic Approach of De-NOx by NH3 on V2O5/WO3/TiO2 Catalysts. 1. Individual Heats of Adsorption of Adsorbed NH3 Species on a Sulfate-Free TiO2 Support Using Adsorption Isobars. J. Phys. Chem. C 2014, 118, 15664-15676.

41. Liu, J.; Meeprasert, J.; Namuangruk, S.; Zha, K.; Li, H.; Huang, L.; Maitarad, P.; Shi, L.; Zhang, D. Facet-Activity Relationship of TiO2 in Fe2O3/TiO2 Nanocatalysts for Selective Catalytic Reduction of NO with NH3: In Situ DRIFTs and DFT Studies. The J. Phys. Chem. C 2017, 121, 4970-4979.

42. Topsoe, N.-Y. Mechanism of the Selective Catalytic Reduction of Nitric Oxide by Ammonia Elucidated by in Situ On-Line Fourier Transform Infrared Spectroscopy. Science 1994, 265, 1217-1219.

43. Tanaka, T.; Teramura, K.; Arakaki, K.; Funabiki, T. Photoassisted NO reduction with NH3 over TiO2 photocatalyst. Chem. Commun. 2002, 2742-2743.

44. Teramura, K.; Tanaka, T.; Funabiki, T. EPR study of photoinduced electron transfer between adsorbent and adsorbed species in photo-SCR with NH3. Chem. Lett. 2003, 32, 1184-1185.

45. Teramura, K.; Tanaka, T.; Yamazoe, S.; Arakaki, K.; Funabiki, T. Kinetic study of photo-SCR with NH3 over TiO2. Appl. Catal., B 2004, 53, 29-36.

46. Yamazoe, S.; Okumura, T.; Teramura, K.; Tanaka, T. Development of the efficient TiO2 photocatalyst in photoassisted selective catalytic reduction of NO with NH3. Catal. Today 2006, 111, 266-270, 10th Japan-Korea Symposium on Catalysis, Matsue, JAPAN, MAY 10-12, 2005. 
47. Yamazoe, S.; Teramura, K.; Hitomi, Y.; Shishido, T.; Tanaka, T. Visible light absorbed $\mathrm{NH} 2$ species derived from $\mathrm{NH} 3$ adsorbed on $\mathrm{TiO} 2$ for photoassisted selective catalytic reduction. J. Phys. Chem. C 2007, 111, 14189-14197.

48. Yamamoto, A.; Mizuno, Y.; Teramura, K.; Shishido, T.; Tanaka, T. Effects of reaction temperature on the photocatalytic activity of photo-SCR of NO with NH3 over a TiO2 photocatalyst. CATALYSIS SCIENCE \& TECHNOLOGY 2013, 3, 1771-1775.

49. Lasek, J.; Yu, Y.-H.; Wu, J. C. S. Removal of NOx by photocatalytic processes. J. Photochem. Photobiol., C 2013, 14, 29-52.

50. Sun, C.; Liu, L.-m.; Selloni, A.; Lu, G. Q. M.; Smith, S. C. Titania-water interactions: a review of theoretical studies. J. Mater. Chem. 2010, 20, 10319.

51. Harris, L. A.; Quong, A. A. Molecular Chemisorption as the Theoretically Preferred Pathway for Water Adsorption on Ideal Rutile TiO2(110). Phys. Rev. Lett. 2004, 086105.

52. Kumar, N.; Kent, P. R. C.; Wesolowski, D. J.; Kubicki, J. D. Modeling water adsorption on rutile (110) using van der waals density functional and DFT+U methods. J. Phys. Chem. C 2013, 117, 23638-23644.

53. Pang, C. L.; Lindsay, R.; Thornton, G. Chemical reactions on rutile TiO2(110). Chem. Soc. Rev. 2008, 37, 2328-2353.

54. Pang, C. L.; Lindsay, R.; Thornton, G. Structure of Clean and Adsorbate-Covered Single-Crystal Rutile TiO2Surfaces. Chem. Rev. 2013, 113, 3887-3948.

55. Lindan, P.; Harrison, N.; Holender, J.; Gillan, M. First-principles molecular dynamics simulation of water dissociation on TiO2 (110). Chem. Phys. Lett. 1996, 261, 246-252.

56. Bates, S.; Kresse, G.; Gillan, M. The adsorption and dissociation of ROH molecules on TiO2(110). Surf. Sci. 1998, 409, 336-349. 
57. Schaub, R.; Thostrup, P.; Lopez, N.; Lægsgaard, E.; Stensgaard, I.; Nørskov, J. K.; Besenbacher, F. Oxygen Vacancies as Active Sites for Water Dissociation on Rutile TiO 2( 110 ). Phys. Rev. Lett. 2001, 87.

58. Lindan, P. J. D.; Zhang, C. Exothermic water dissociation on the rutileTiO2(110)surface. Phys. Rev. B 2005, 72.

59. Kowalski, P. M.; Meyer, B.; Marx, D. Composition, structure, and stability of the rutileTiO2(110)surface: Oxygen depletion, hydroxylation, hydrogen migration, and water adsorption. Phys. Rev. B 2009, 79.

60. Henderson, M. A. An HREELS and TPD study of water on TiO2(110): the extent of molecular versus dissociative adsorption. Surf. Sci. 1996, 355, 151-166.

61. Krischok, S.; Höfft, O.; Günster, J.; Stultz, J.; Goodman, D.; Kempter, V. H2O interaction with bare and Li-precovered TiO2: studies with electron spectroscopies (MIES and UPS(HeI and II)). Surf. Sci. 2001, 495, 8-18.

62. Ketteler, G.; Yamamoto, S.; Bluhm, H.; Andersson, K.; Starr, D. E.; Ogletree, D. F.; Ogasawara, H.; Nilsson, A.; Salmeron, M. The Nature of Water Nucleation Sites on TiO2(110) Surfaces Revealed by Ambient Pressure X-ray Photoelectron Spectroscopy. J. Phys. Chem. C 2007, 111, 8278-8282.

63. Hugenschmidt, M. B.; Gamble, L.; Campbell, C. T. The interaction of H2O with a TiO2(110) surface. Surf. Sci. 1994, 302, 329-340.

64. ichi Ishibashi, K.; Fujishima, A.; Watanabe, T.; Hashimoto, K. Quantum yields of active oxidative species formed on $\mathrm{TiO} 2$ photocatalyst. J. Photochem. Photobiol., A 2000, 134, 139-142.

65. Xiang, Q.; Yu, J.; Wong, P. K. Quantitative characterization of hydroxyl radicals produced by various photocatalysts. J. Colloid Interface Sci. 2011, 35\%, 163-167. 
66. Yan, J.; Wu, G.; Guan, N.; Li, L.; Li, Z.; Cao, X. Understanding the effect of surface/bulk defects on the photocatalytic activity of TiO2: anatase versus rutile. Phys. Chem. Chem. Phys. 2013, 15, 10978.

67. Diebold, U. The surface science of titanium dioxide. Surf. Sci. Rep. 2003, 48 .

68. Reuter, K.; Scheffler, M. Composition, structure, and stability of RuO2(110) as a function of oxygen pressure. Phys. Rev. B 2001, 65.

69. Reuter, K.; Stampf, C.; Scheffler, M. Handbook of Materials Modeling; Springer Nature, $2005 ;$ pp 149-194.

70. Calle-Vallejo, F.; Koper, M. T. First-principles computational electrochemistry: Achievements and challenges. Electrochim. Acta 2012, 84, 3-11.

71. Nørskov, J. K.; Rossmeisl, J.; Logadottir, A.; Lindqvist, L.; Kitchin, J. R.; Bligaard, T.; Jónsson, H. Origin of the Overpotential for Oxygen Reduction at a Fuel-Cell Cathode. J. Phys. Chem. B 2004, 108, 17886-17892.

72. Man, I. C.; Su, H.-Y.; Calle-Vallejo, F.; Hansen, H. A.; Martínez, J. I.; Inoglu, N. G.; Kitchin, J.; Jaramillo, T. F.; Nørskov, J. K.; Rossmeisl, J. Universality in Oxygen Evolution Electrocatalysis on Oxide Surfaces. ChemCatChem 2011, 3, 1159-1165.

73. Peterson, A. A.; Abild-Pedersen, F.; Studt, F.; Rossmeisl, J.; Nørskov, J. K. How copper catalyzes the electroreduction of carbon dioxide into hydrocarbon fuels. Energy Environ. Sci. 2010, 3, 1311.

74. Skulason, E.; Bligaard, T.; Gudmundsdóttir, S.; Studt, F.; Rossmeisl, J.; AbildPedersen, F.; Vegge, T.; Jónsson, H.; Nørskov, J. K. A theoretical evaluation of possible transition metal electro-catalysts for N 2 reduction. Phys. Chem. Chem. Phys. 2012, $14,1235-1245$. 
75. Wellendorff, J.; Lundgaard, K. T.; Møgelhøj, A.; Petzold, V.; Landis, D. D.; Nørskov, J. K.; Bligaard, T.; Jacobsen, K. W. Density functionals for surface science: Exchange-correlation model development with Bayesian error estimation. Phys. Rev. B 2012, 85, 235149-235149.

76. Hellman, A.; Wang, B. First-Principles View on Photoelectrochemistry: WaterSplitting as Case Study. Inorganics 2017, 5, 37.

77. de Dompablo, M. E. A.; Morales-García, A.; Taravillo, M. DFT+U calculations of crystal lattice, electronic structure, and phase stability under pressure of $\mathrm{TiO} 2$ polymorphs. J. Chem. Phys. 2011, 135, 054503.

78. Zhu, D.; Zhang, L.; Ruther, R. E.; Hamers, R. J. Photo-illuminated diamond as a solidstate source of solvated electrons in water for nitrogen reduction. Nat. Mater. 2013, 12, 836-841.

79. Vettraino, M.; Trudeau, M.; Lo, A. Y. H.; Schurko, R. W.; Antonelli, D. RoomTemperature Ammonia Formation from Dinitrogen on a Reduced Mesoporous Titanium Oxide Surface with Metallic Properties. J. Am. Chem. Soc. 2002, 124, 9567-9573.

80. Ali, M.; Zhou, F.; Chen, K.; Kotzur, C.; Xiao, C.; Bourgeois, L.; Zhang, X.; MacFarlane, D. R. Nanostructured photoelectrochemical solar cell for nitrogen reduction using plasmon-enhanced black silicon. Nat. Commun. 2016, 7, 11335.

81. Nozik, A. J.; Memming, R. Physical Chemistry of Semiconductor-Liquid Interfaces. The J. Phys. Chem. 1996, 100, 13061-13078.

82. Montoya, J. H.; Tsai, C.; Vojvodic, A.; Nørskov, J. K. The challenge of electrochemical ammonia synthesis: A new perspective on the role of nitrogen scaling relations. ChemSusChem 2015, 8, 2180-2186. 
83. Singh, A. R.; Rohr, B. A.; Schwalbe, J. A.; Cargnello, M.; Chan, K.; Jaramillo, T. F.; Chorkendorff, I.; Nørskov, J. K. Electrochemical Ammonia Synthesis-The Selectivity Challenge. ACS Catal. 2017, 7, 706-709.

84. Emmett, P. H.; Brunauer, S. THE ADSORPTION OF NITROGEN BY IRON SYNTHETIC AMMONIA CATALYSTS. J. Am. Chem. Soc. 1933, 55, 1738-1739.

85. Ertl, G.; Grunze, M.; Weiss, M. Chemisorption of N2 on an Fe(100) surface. J. Vac. Sci. Technol. 1976, 13, 314-317.

86. Spencer, N. D.; Schoonmaker, R. C.; Somorjai, G. A. Structure sensitivity in the iron single-crystal catalysed synthesis of ammonia. Nature 1981, 294, 643-644.

87. Honkala, K. Ammonia Synthesis from First-Principles Calculations. Science 2005, 307, $555-558$.

88. Vojvodic, A.; Medford, A. J.; Studt, F.; Abild-Pedersen, F.; Khan, T. S.; Bligaard, T.; Nørskov, J. K. Exploring the limits: A low-pressure, low-temperature Haber-Bosch process. Chem. Phys. Lett. 2014, 598, 108-112.

89. Karlberg, G. S.; Rossmeisl, J.; Nørskov, J. K. Estimations of electric field effects on the oxygen reduction reaction based on the density functional theory. Phys. Chem. Chem. Phys. 2007, 9, 5158.

90. He, Z.-D.; Hanselman, S.; Chen, Y.-X.; Koper, M. T. M.; Calle-Vallejo, F. Importance of Solvation for the Accurate Prediction of Oxygen Reduction Activities of Pt-Based Electrocatalysts. J. Phys. Chem. Lett. 2017, 8, 2243-2246.

91. van der Ham, C. J. M.; Koper, M. T. M.; Hetterscheid, D. G. H. Challenges in reduction of dinitrogen by proton and electron transfer. Chem. Soc. Rev. 2014, 43, 5183.

92. Abghoui, Y.; Garden, A. L.; Howalt, J. G.; Vegge, T.; Skúlason, E. Electroreduction of 
$\mathrm{N}_{2}$ to Ammonia at Ambient Conditions on Mononitrides of $\mathrm{Zr}, \mathrm{Nb}, \mathrm{Cr}$, and V: A DFT Guide for Experiments. ACS Catalysis 2016, 6, 635-646.

93. Yates, J. T.; Szabó, A.; Henderson, M. A. Structure-Activity and Selectivity Relationships in Heterogeneous Catalysis, Proceedings of the ACS Symposium on StructureActivity Relationships in Heterogeneous Catalysis; Elsevier, 1991; pp 273-290.

94. McFarland, E. W.; Metiu, H. Catalysis by Doped Oxides. Chem. Rev. 2013, 113, 43914427.

95. Hobiger, G.; Herzig, P.; Eibler, R.; Schlapansky, F.; Neckel, A. The influence of titanium and oxygen vacancies on the chemical bonding in titanium oxide. J. Phys.: Condens. Matter 1990, 2, 4595-4612.

96. Goldsmith, B. R.; Sanderson, E. D.; Bean, D.; Peters, B. Isolated catalyst sites on amorphous supports: A systematic algorithm for understanding heterogeneities in structure and reactivity. J. Chem. Phys. 2013, 138, 204105.

97. Ranjit, K.; Viswanathan, B. Photocatalytic reduction of nitrite and nitrate ions to ammonia on M/TiO2 catalysts. J. Photochem. Photobiol., A 1997, 108, 73-78.

98. Kobwittaya, K.; Sirivithayapakorn, S. Photocatalytic reduction of nitrate over TiO2 and Ag-modified TiO2. J. Saudi Chem Soc. 2014, 18, 291-298.

99. Kominami, H.; Gekko, H.; Hashimoto, K. Photocatalytic disproportionation of nitrite to dinitrogen and nitrate in an aqueous suspension of metal-loaded titanium(iv) oxide nanoparticles. Phys. Chem. Chem. Phys. 2010, 12, 15423.

100. Shand, M.; Anderson, J. A. Aqueous phase photocatalytic nitrate destruction using titania based materials: routes to enhanced performance and prospects for visible light activation. Catal.: Sci. Technol. 2013, 3, 879. 
101. Lozovskii, A. V.; Stolyarova, I. V.; Prikhod'ko, R. V.; Goncharuk, V. V. Research of photocatalytic activity of the $\mathrm{Ag} / \mathrm{TiO} 2$ catalysts in the reduction reaction of nitrateions in aqueous media. J. Water Chem. Technol. 2009, 31, 360-366.

102. Pollema, C. H.; Milosavljević, E. B.; Hendrix, J. L.; Solujić, L.; Nelson, J. H. Photocatalytic oxidation of aqueous ammonia (ammonium ion) to nitrite or nitrate at $\mathrm{TiO} 2$ particles. Monatshefte fr Chemie Chemical Monthly 1992, 123, 333-339.

103. Wang, A.; Edwards, J. G.; Davies, J. A. Photooxidation of aqueous ammonia with titania-based heterogeneous catalysts. Sol. Energy 1994, 52, 459-466.

104. Kominami, H.; Kitsui, K.; Ishiyama, Y.; Hashimoto, K. Simultaneous removal of nitrite and ammonia as dinitrogen in aqueous suspensions of a titanium(iv) oxide photocatalyst under reagent-free and metal-free conditions at room temperature. $R S C A d v \mathbf{2 0 1 4}, 4$, $51576-51579$.

105. Urabe, K. Isotopic equilibration of nitrogen on potassium-promoted transition metal catalysts. J. Catal. 1978, 54, 436-438.

106. Giannozzi, P. et al. QUANTUM ESPRESSO: a modular and open-source software project for quantum simulations of materials. J. Phys.: Condens. Matter 2009, 21, 395502 (19pp).

107. Bahn, S. R.; Jacobsen, K. W. An object-oriented scripting interface to a legacy electronic structure code. Comput. Sci. Eng. 2002, 4, 56-66.

108. Monkhorst, H. J.; Pack, J. D. Special points for Brillouin-zone integrations. Phys. Rev. B 1976, 13, 5188-5192.

109. Bengtsson, L. Dipole correction for surface supercell calculations. Phys. Rev. B 1999, 59, 12301-12304. 
110. Bredow, T.; Giordano, L.; Cinquini, F.; Pacchioni, G. Electronic properties of rutile TiO2 ultrathin films: Odd-even oscillations with the number of layers. Phys. Rev. B Condens. Matter and Mater. Phys. 2004, 70, 1-6.

111. Patel, M.; Sanches, F. F.; Mallia, G.; Harrison, N. M. A quantum mechanical study of water adsorption on the (110) surfaces of rutile $\mathrm{SnO} 2$ and $\mathrm{TiO} 2$ : investigating the effects of intermolecular interactions using hybrid-exchange density functional theory. Phys. Chem. Chem. Phys. 2014, 16, 21002-21015.

112. Wellendorff, J.; Silbaugh, T. L.; Garcia-Pintos, D.; Nørskov, J. K.; Bligaard, T.; Studt, F.; Campbell, C. T. A benchmark database for adsorption bond energies to transition metal surfaces and comparison to selected DFT functionals. Surf. Sci. 2015, $640,36-44$.

113. Li, W.-K.; Gong, X.-Q.; Lu, G.; Selloni, A. Different Reactivities of TiO 2 Polymorphs: Comparative DFT Calculations of Water and Formic Acid Adsorption at Anatase and Brookite TiO 2 Surfaces. J. Phys. Chem. C 2008, 112, 6594-6596.

114. Zheng, T.; Wu, C.; Chen, M.; Zhang, Y.; Cummings, P. T. A DFT study of water adsorption on rutile TiO2 (110) surface: The effects of surface steps. The J. Chem. Phys. 2016, 145, 044702.

115. Morgan, B. J.; Watson, G. W. A DFT + U description of oxygen vacancies at the TiO2 rutile (110) surface. Surf. Sci. 2007, 601, 5034-5041.

116. Landmann, M.; Rauls, E.; Schmidt, W. G. The electronic structure and optical response of rutile, anatase and brookite TiO 2. J. Phys.: Condens. Matter 2012, 24, 195503.

117. Deák, P.; Aradi, B.; Frauenheim, T. Polaronic effects in TiO 2 calculated by the HSE06 hybrid functional: Dopant passivation by carrier self-trapping. Phys. Rev. B 2011, 83. 
118. Jauho, T. S.; Olsen, T.; Bligaard, T.; Thygesen, K. S. Improved description of metal oxide stability: Beyond the random phase approximation with renormalized kernels. Phys. Rev. B 2015, 92.

119. Walker, E.; Ammal, S. C.; Terejanu, G. A.; Heyden, A. Uncertainty Quantification Framework Applied to the Water-Gas Shift Reaction over Pt-Based Catalysts. The J. Phys. Chem. C 2016, 120, 10328-10339.

120. Medford, A. J.; Vojvodic, A.; Hummelshøj, J. S.; Voss, J.; Abild-Pedersen, F.; Studt, F.; Bligaard, T.; Nilsson, A.; Nørskov, J. K. From the Sabatier principle to a predictive theory of transition-metal heterogeneous catalysis. J. Catal. 2015, 328, 36-42. 


\section{For Table of Contents Use Only.}

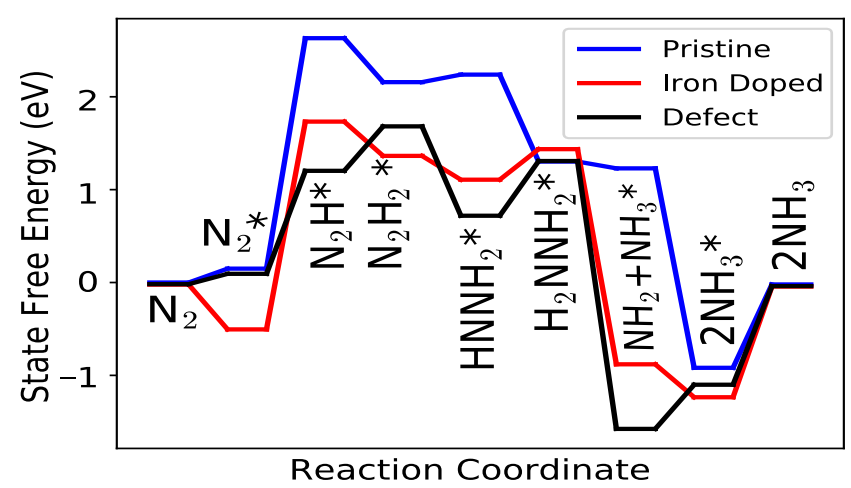

Figure 11: Energetics of photocatalytic nitrogen fixation over rutile (110) titania active sites. Photocatalysis provides a route to nitrogen fixation at atmospheric conditions and sustainable fertilizer production. 